\title{
Survival of the $\mathrm{mm}-\mathrm{cm}$ size grain population observed in protoplanetary disks ${ }^{\star}$
}

\author{
F. Brauer ${ }^{1}$, C. P. Dullemond ${ }^{1}$, A. Johansen ${ }^{1}$, Th. Henning ${ }^{1}$, H. Klahr ${ }^{1}$, and A. Natta ${ }^{2}$ \\ 1 Max-Planck-Institut für Astronomie, Königstuhl 17, 69117 Heidelberg, Germany \\ e-mail: brauer@mpia.de \\ 2 Osservatorio Astrofisico di Arcetri, Largo Enrico Fermi 5, 50125 Firenze, Italy \\ e-mail: natta@arcetri.astro.it
}

Received 4 December 2006 / Accepted 6 April 2007

\begin{abstract}
Millimeter interferometry provides evidence for the presence of $\mathrm{mm}$ to $\mathrm{cm}$ size "pebbles" in the outer parts of disks around pre-mainsequence stars. The observations suggest that large grains are produced relatively early in disk evolution $(<1 \mathrm{Myr})$ and remain at large radii for longer periods of time ( 5 to $10 \mathrm{Myr}$ ). Simple theoretical estimates of the radial drift time of solid particles, however, imply that they would drift inward over a time scale of less than $0.1 \mathrm{Myr}$. In this paper, we address this conflict between theory and observation, using more detailed theoretical models, including the effects of sedimentation, collective drag forces and turbulent viscosity. We find that, although these effects slow down the radial drift of the dust particles, this reduction is not sufficient to explain the observationally determined long survival time of $\mathrm{mm} / \mathrm{cm}$-sized grains in protoplanetary disks. However, if for some reason the gas to dust ratio in the disk is reduced by at least a factor of 20 from the canonical value of 100 (for instance through photoevaporation of the gas), then the radial drift time scales become sufficiently large to be in agreement with observations.
\end{abstract}

Key words. accretion, accretion disks - stars: circumstellar matter - stars: formation - stars: pre-main sequence - infrared: stars stars: planetary systems: protoplanetary disks

\section{Introduction}

Millimeter and sub-millimeter observations have shown the presence of large amounts of millimeter to centimeter-sized grains in the outer regions ( $100 \mathrm{AU})$ of disks around Herbig Ae and T Tauri stars (Testi et al. 2003; Rodmann et al. 2006; Wilner et al. 2005; Natta et al. 2007). The presence of these grains, which are much larger than the grains typically found in the interstellar medium, is often regarded as evidence that the first steps of planet formation are taking place in these disks. The presence of such large grains, however, also poses a major problem. According to simple theoretical considerations, grains of this size undergo a rapid radial drift (Whipple 1972; Weidenschilling 1977), which causes them to disappear from the outer disk in a very short time (Takeuchi \& Lin 2005; Klahr \& Bodenheimer 2006; Alexander \& Armitage 2007). However the typical age of protoplanetary disks that are observed at millimeter wavelengths is a few million years, which is much longer than this radial drift time scale. Takeuchi \& Lin propose that either the entire grain growth process is slow or that the grains are the collision products of a population of even larger bodies $(\gtrsim 10 \mathrm{~m})$. The second explanation requires that in addition to the grain population that is observed at $\mathrm{mm}$ wavelengths, there is a population of smaller/larger bodies which act as a reservoir of solid material from which $\mathrm{mm} / \mathrm{cm}$-sized grains are continuously produced. The problem is that if the drift time scale is, for example, 20 times shorter than the disk life time, this reservoir of larger bodies must contain at least 20 times more mass than the observed dust mass. If it is assumed that the particle size

* Appendices are only available in electronic form at http://www. aanda.org distribution follows a powerlaw then the total mass of the disk and the minimum upper particle size of this distribution can directly be calculated from the slope of the mm flux of the protoplanetary disk. This analysis shows that the amount of dust responsible for the millimeter fluxes of these disks is in many cases already very high, of the order of $10^{-3} M_{\odot}$ or even higher (Testi et al. 2003; Natta et al. 2004; Wilner et al. 2005; Rodmann et al. 2006; Rodmann 2006). A 20 times more massive reservoir of larger (non-observable) bodies is then clearly unrealistic. These arguments suggest that perhaps the standard theoretical estimate of the radial drift may be not applicable.

Interestingly, a related drift problem is present for the theory of planetesimal formation. The drift problem for $\mathrm{mm} / \mathrm{cm}$-sized particles at $100 \mathrm{AU}$, that will be investigated in this paper, similarly shows up for meter-sized particles at $1 \mathrm{AU}$. The radial drift of these large bodies in the inner parts of the disk is so high that they should drift into the evaporation zone over time scales of $\sim 10^{2} \mathrm{yr}$. This is one of the fundamental and unresolved problems of planet formation (Dominik et al. 2007). In that sense, the $\mathrm{cm}$ problem at $100 \mathrm{AU}$ is a proxy for the meter problem at $1 \mathrm{AU}$, and figuring out a solution at $100 \mathrm{AU}$ may give important clues to what happens at $1 \mathrm{AU}$.

The goal of this paper is to study the radial drift of mm/cmsized particles in more detail. We will investigate the magnitude of the drift problem, and which effects might keep the grains for a few Myr in the outer parts of the disk. To address this issue we will proceed in steps. In the first step we will review the radial drift of individual particles (Whipple 1972; Weidenschilling 1977). This section will show that the drift time scale of such particles is orders of magnitude smaller than the age of the disks observed (5 to $10 \mathrm{Myr}$ ). In a second step, we explore the 
possibility that collective effects of the dust might slow down the drift. Collective effects take place when the dust settles into a thin midplane layer (Dubrulle et al. 1995; Garaud et al. 2004; Schräpler \& Henning 2004). This process increases locally the dust-to-gas ratio, and the dynamics of the dust starts to affect the gas motion (Nakagawa et al. 1986; Johansen et al. 2006a). This may, in turn, reduce the relative velocities between the dust and the gas, and hence reduce the head wind that causes radial drift. We will investigate the magnitude of the reduction and if thin midplane layers yield a possibility to increase the drift time scales to some Myr. In the third step we improve on these calculations by including vertical angular momentum exchanges in the disk through turbulent viscosity. Finally, we speculate on other potential ways in which $\mathrm{mm} / \mathrm{cm}$-sized grains could be prevented from drifting inward on a time scale shorter than the life time of the disk: particle trapping in vortices and gas pressure maxima (Barge \& Sommeria 1995; Klahr \& Henning 1997; Fromang \& Nelson 2005; Johansen et al. 2006b), spiral arms (Rice et al. 2004) and photoevaporation of the gas leaving the dust behind (Alexander et al. 2006).

Over the last decades various papers determined the radial drift of dust particles and the structure and dynamics of thin midplane dust layers. In particular the latter problem has attracted much attention, but for an entirely different reason than ours: gravitational instabilities in thin midplane dust layers are thought to be a possible origin of planetesimals (Goldreich \& Ward 1973). A lively debate has since appeared about the viability of this concept, spurring various papers including models of midplane dust layers (Weidenschilling 1980; Sekiya 1998; Weidenschilling 2006; Cuzzi \& Weidenschilling 2006; Youdin \& Shu 2002; Youdin \& Chiang 2004; Johansen et al. 2006a). The richness of this literature gives an indication of the complexity of the problem. Hence, due to this complexity only a sub-set of the possible physical effects are considered in these dust layer models. In particular the collective effects of the dust and the effects of vertical and radial viscosity have not been studied yet in combination. Therefore, another goal of this paper is to present a model of dense dust midplane layers that include a multitude of physical effects, albeit still in the form of a 1D vertical model.

In this paper we do not consider dust particle coagulation. Larger particles in protostellar disks beyond sizes that can be found in the interstellar medium form by collisional sticking due to relative motions of the dust (Beckwith et al. 2000; Blum \& Wurm 2000). However, at $100 \mathrm{AU}$ in the disk the dust particles rapidly drift inwards before they can even grow to sizes that are discussed this paper (Brauer et al., in prep.). In the present paper we will ignore the issue of the formation of these $\mathrm{mm} / \mathrm{cm}$ size grains and assume that all the dust is in the form of grains of a given size (a "monodisperse" size distribution). We will then investigate whether these grains can remain in the outer regions of the disk for a sufficiently long time.

The structure of this paper is as follows. In Sect. 2 we discuss the radial drift of the dust and its radial drift time scales. Section 3 includes other possibilities to increase the drift time scales. A detailed discussion of the theoretical background is given in the Appendix A.

\section{Radial drift of the dust}

We consider a T Tauri star with a stellar mass of $1 M_{\odot}$, a stellar radius of $2.5 R_{\odot}$ and a surface temperature of $4000 \mathrm{~K}$. We assume that the disk around this pre-main sequence star has an inner and an outer radius of $0.03 \mathrm{AU}$ and $150 \mathrm{AU}$ (Rodmann et al. 2006), respectively. The mass of the disk $M_{\text {disk }}$ is a free parameter of our
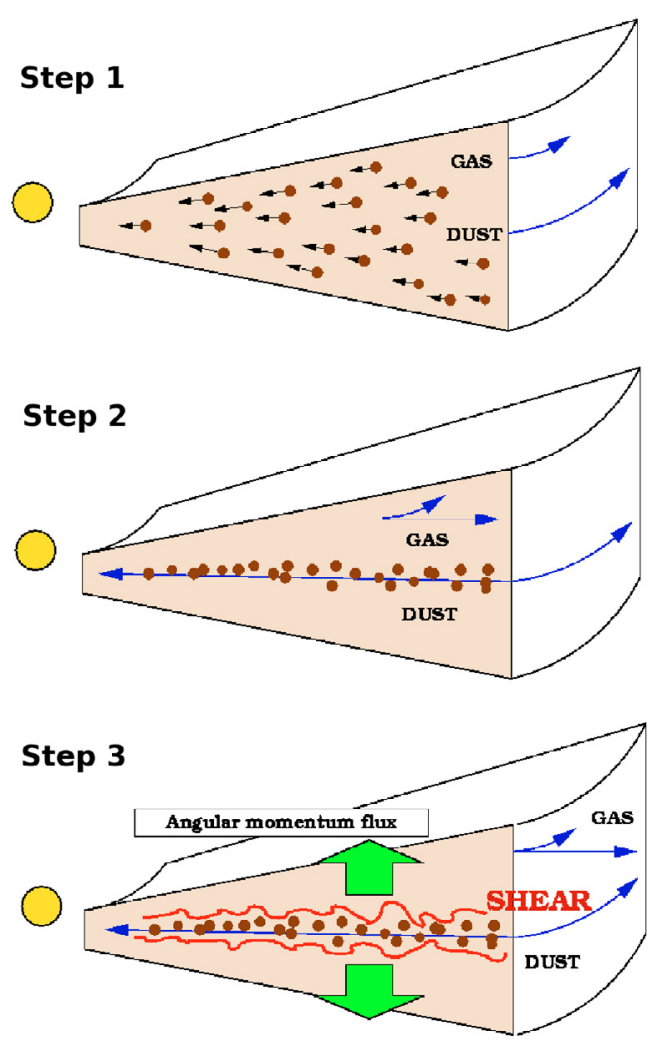

Fig. 1. This figure shows the three scenarios we will investigate in this paper. In step 1 we consider the drift of individual particles. In step 2 we investigate the influence of collective effects on the particle drift. These effects become of importance if the particles settle into a thin midplane layer. Finally, in step 3 we will investigate what happens if we include turbulent viscosity in our simulations. In this case, angular momentum is exchanged between the dusty midplane layer and the gaseous layers above the midplane. The horizontal arrows indicate the radial velocity of gas and dust. The curved arrows indicate the azimuthal velocities.

model. We assume that the disk is passive and irradiated by the central star under an angle of $0.05 \mathrm{rad}$. At a disk radius of $1 \mathrm{AU}$ these values imply a midplane temperature of $200 \mathrm{~K}$ assuming that the disk is isothermal in the vertical direction. Moreover, we will consider a turbulent disk. The amount of turbulence in the disk is described by the turbulent $\alpha$ parameter (Shakura \& Sunyaev 1973), which is a free parameter of our model.

Now, we will calculate the radial drift of the dust and the radial drift time scales. In order to demonstrate the physics behind the calculations and its implications on the radial velocities we will proceed in certain steps. In every step more effects are included to demonstrate the influence on the drift velocities (cf. Fig. 1).

1. The first subsection addresses the drift of individual particles in a gaseous disk. These results are valid when the dust and the gas are well mixed.

2. However, under certain conditions (low-turbulence disks, large dust particles), the dust sediments so close to the midplane that the density of the dust exceeds the density of the gas. In that case collective effects of the dust come into play, which is the topic of the second subsection.

3. Finally, in the last subsection, we also include the effect of vertical angular momentum exchange between the dust midplane layer and the gaseous layers above the midplane.

We are primarily interested in drift time scales needed to keep $\mathrm{mm}$ to $\mathrm{cm}$ size particles in the outer parts of the disk, hence, we 


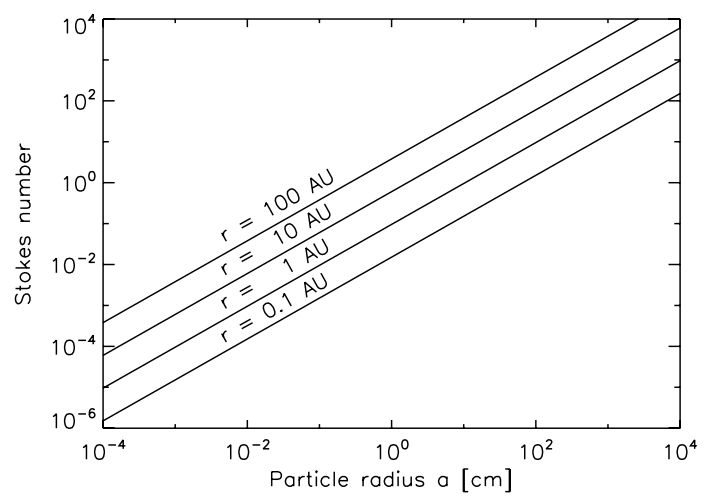

Fig. 2. The Stokes number St as a function of particle radius $a$ at different radii in the disk. In this calculation the solid particle density of the dust is $1.6 \mathrm{~g} / \mathrm{cm}^{3}$ and the stellar mass is $1 M_{\odot}$. The innner and the outer radius of the disk are $0.03 \mathrm{AU}$ and $150 \mathrm{AU}$, respectively. The disk mass is $10^{-2} M_{\odot}$.

will calculate characteristic drift times at the very end of every of these three subsections. We will see how these time scales are affected by including more and more effects.

\subsection{Step 1 - Radial drift of individual particles}

\subsubsection{Equations}

The fundamental cause for inward drift of the dust is the difference in velocity between gas and dust. While the dust moves with Keplerian velocity the gas moves slightly sub-Keplerian. This is due to the fact that the gas is not only affected by the gravitational and the centrifugal force but additionally feels a radial pressure force that does not act on dust particles. This extra force is caused by the decrease of gas pressure in the radial direction. Since this force, which exclusively acts on the gas, partly compensates gravitation, the gas moves slower than Kepler speed and therefore slower than any dust particle in the disk. Hence, the dust particle feels a continuous headwind from the gas. This headwind causes the dust particle to lose its angular momentum and spiral inward.

Whipple (1972) formulated the first equations for the radial drift of very small and very large particles. Weidenschilling (1977) later derived a set of equations with a general drag force to calculate the radial drift of solid particles of any size. We will formulate all equations in the dimensionless Stokes number formulation. The Stokes number can be regarded as a measure of grain size (see Fig. 2). The full definition is given in Appendix A.4. In terms of this dimensionless formulation, these equations aquire the form ${ }^{1}$

$0=\frac{w_{\varphi}}{\mathrm{St}}+\frac{w_{r}}{2}$,

$0=w_{\varphi}^{2}+v_{\mathrm{N}} w_{\varphi}+\frac{w_{r}^{2}}{4}$.

The variables $w_{r}$ and $w_{\varphi}$ denote the radial and azimuthal velocity of the dust, respectively. The form of the drag law is implicitly included in the Stokes number. The quantity $v_{\mathrm{N}}$ is the velocity by which the gas moves azimuthally slower than Keplerian velocity $V_{\mathrm{k}}$, i.e. $v_{\text {gas }}=V_{\mathrm{k}}-v_{\mathrm{N}}$. The velocity $v_{\mathrm{N}}$ will also turn out to be the maximum radial drift velocity of the dust. We will take $v_{\mathrm{N}}$ from here on as our "standard velocity" scale (cf. Eq. (A.22))

1 The physical meaning of all variables is listed at the very end of the paper.

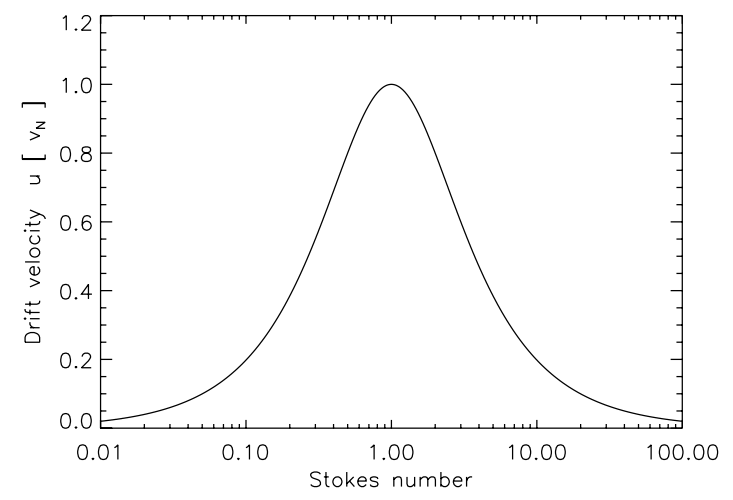

Fig. 3. The radial drift velocity of individual particles $w_{r}$ in units of $v_{\mathrm{N}}$ as a function of Stokes number.

apart from the Keplerian velocity $V_{\mathrm{k}}$. For our disk model the quantity $v_{\mathrm{N}}$ is given by (Weidenschilling 1977; Nakagawa et al. 1986)

$v_{\mathrm{N}}=1.28 \frac{c_{\mathrm{s}}^{2}}{V_{\mathrm{k}}}$

In this expression $c_{\mathrm{s}}$ denotes the isothermal sound speed. The number 1.28 is due to the specifications of our disk model (cf. App. A.6). Note that the velocity scale $v_{\mathrm{N}}$ does not depend on the disk mass. In our disk model this quantity is also not dependent on the location $r$ in the disk.

The set of Eqs. (1) is generally difficult to solve and only numerical methods provide information about the drift velocity. However, in some cases the situation simplifies. If the Stokes number is not dependent on the particle velocity, Eqs. (1) can be solved analytically. Assuming this independency a straightforward calculation yields

$w_{r}=\frac{2}{\mathrm{St}+\frac{1}{\mathrm{St}}} v_{\mathrm{N}}$.

This equation directly shows that the drift velocity has a maximum when the Stokes number is unity and the maximal drift velocity is $v_{\mathrm{N}}$. The drift velocity as a function of Stokes number is shown in Fig. 3.

\subsubsection{Drift time scales for individual particles}

We will now investigate the radial drift times of individual particles in the outer parts of the disk. More specifically we are interested in the conditions on particle radius and particle porosity that provide time scales larger than a few Myr.

The drift timescale $\tau_{\text {drift }}$ equals

$\tau_{\text {drift }}=\frac{r}{w_{r}}$,

which should correspond to the age $\tau_{\text {age }}$ of the disks observed, thus a few Myr. Throughout the paper we focus on the radius of $r=100$ AU. To find the critical Stokes numbers for which the radial drift time scale equals the age of the disk, we replace $\tau_{\text {drift }}$ in Eq. (4) by $\tau_{\text {age }}$. We then insert Eqs. (3) into (4), and solve for St. This yields two critical Stokes numbers:

$\mathrm{St}_{ \pm}=\frac{\tau_{\mathrm{age}} v_{\mathrm{N}}}{r} \pm \sqrt{\left(\frac{\tau_{\mathrm{age}} v_{\mathrm{N}}}{r}\right)^{2}-1}$

The interpretation of these two numbers is the following: if the Stokes number of the dust particle falls into the interval 
$\left[\mathrm{St}_{-}, \mathrm{St}_{+}\right]$, then the drift timescale is shorter than $\tau_{\text {age }}$. If it falls outside of this interval, then the drift time scale is long enough that these particles can be observed in the protoplanetary disk of age $\tau_{\text {age }}$. Since the Stokes number interval is a rather abstract depiction we reformulated it into a particle radius interval with a similar meaning.

The region of too short time scales at $100 \mathrm{AU}$ is shown in Fig. 4 as a function of disk mass $M_{\text {disk }}$ and surface density $\Sigma$. In this diagram we applied a dust material density ${ }^{2} \rho_{\mathrm{s}}=1.6 \mathrm{~g} \mathrm{~cm}^{-3}$, a Kepler frequency $\Omega_{\mathrm{k}}=10^{-10} / \mathrm{s}$ and $c_{\mathrm{s}}=2.6 \times 10^{4} \mathrm{~cm} \mathrm{~s}^{-1}$ (corresponding to a temperature of $20 \mathrm{~K}$ ). We take as the age of the $\operatorname{disk} \tau_{\text {age }}=2$ Myr. The maximum radial drift velocity at this location is $v_{\mathrm{N}}=60 \mathrm{~m} \mathrm{~s}^{-1}$. We will make use of these values at all times in this paper unless otherwise noted. The two Stokes numbers $\mathrm{St}_{ \pm}$that are implied by these values are $\mathrm{St}_{-}=0.002$ and $\mathrm{St}_{+}=474$, corresponding to the lower and upper edge of the grey zone in Fig. 4 respectively.

The figure shows that the particle radius interval in which the time scale of individual particles is shorter than 2 Myr ranges over more than 5 orders of magnitude in radius. Particles ranging from $\mathrm{mm}$ to $\mathrm{cm}$ in size are completely included in this region independent of disk mass. The drift time scale of submillimeter particles may exceed $\tau_{\text {age }}$ when the disk mass is higher than $0.2 M_{\star}$.

The Stokes number as the crucial value for radial drift is not only affected by particle radius but also by particle properties like porosity or fractal growth (Kempf et al. 2000). This effect of noncompact growth may be considered by introducing the filling factor of the particle $f$ defined by $m_{\mathrm{p}}=V_{\mathrm{p}} \rho_{\mathrm{s}} f$, where $m_{\mathrm{p}}$ and $V_{\mathrm{p}}$ are the mass and the volume of the particle, respectively. In Fig. 4 we also calculated the critical particle radius interval for a filling factor of $f=10^{-1}$ (dotted lines).

The lower filling factor shifts the critical particle radius interval towards higher particle radii. The drift time scale of mm size particles exceeds 2 Myr when disk masses higher then $0.2 M_{\star}$ are considered. For $\mathrm{cm}$ size particles the time scale never exceeds 2 Myr. For filling factors lower than $10^{-3}$ the drift time scales of $\mathrm{mm}$ and $\mathrm{cm}$ size particles exceed $2 \mathrm{Myr}$ for any disk mass higher than $10^{-3} M_{\star}$. However, particles of mass $1 \mathrm{~g}$ and filling factors of $10^{-3}$ would imply a particle diameter of $5 \mathrm{~cm}$. Since this particle size falls into the regime where compaction is thought to occur (Blum \& Wurm 2000) this filling factor represents an unlikely case.

\subsection{Step $2-$ Collective effects}

The scope of the previous subsection can be expanded by including the back-reactions from the dust to the gas. We do no longer consider a single particle, but include how the entire swarm of dust particles can affect the gas motion. The modified gas motion has the effect of reducing the rate by which the gas extracts angular momentum from the dust, and thereby reduces the radial drift of the dust. Such collective effects play the strongest role for low $\alpha$-values so that a thin midplane dust layer can form in which the dust density is high. This scenario of reduction of radial drift was described by Nakagawa et al. (1986).

The necessity to take this additional effect into account may be illustrated by regarding the following extreme scenario. We consider a hypothetical disk in which the dust density is much higher than the gas density. In such a dust-dominated disk the dust is hardly influenced by the gas. The gas, which tends to move sub-Keplerian, is dragged along with the dust since it feels

\footnotetext{
$210 \%$ silicate, $30 \%$ carbonaceous material and $60 \%$ ice.
}

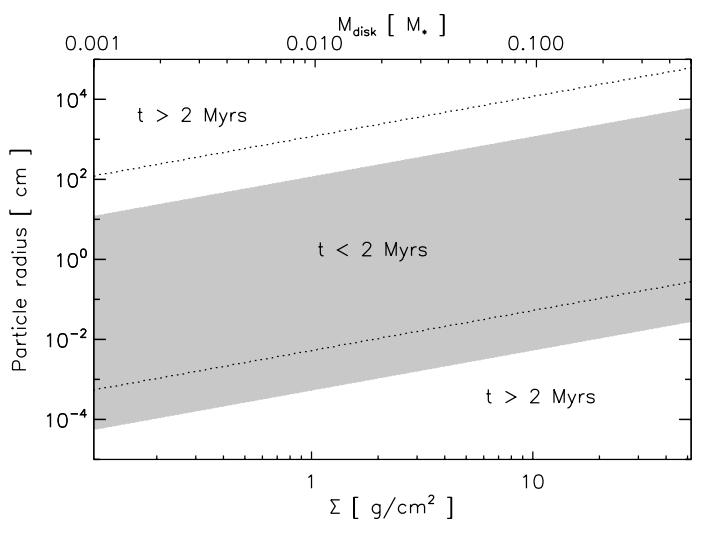

Fig. 4. The particle radius interval in which the individual drift time scale at $r=100$ AU becomes shorter than $2 \mathrm{Myr}$ as a function of surface density or equivalently disk mass (shaded region). The disk mass is computed from the surface density using Eq. (A.21). To illustrate the effect of noncompact growth we also calculated the interval for a filling factor of $10^{-1}$ (dotted lines).

a continuous tailwind of dust particles. Therefore, the gas perpetually gains angular momentum from the dust and spirals outward. The radial drift of the dust is negligible because the dustto-gas ratio is much higher than 1 . This is the reverse situation of the case described in Sect. 2.1. In general, though, we neither have a perfectly dust-dominated nor gas-dominated situation. We then have to solve the following set of equations (Nakagawa et al. 1986)

$0=2 \Omega_{\mathrm{k}} u_{\varphi}-A \rho_{\mathrm{d}}\left(u_{r}-w_{r}\right)-\frac{1}{\rho_{\mathrm{g}}} \partial_{r} p_{\mathrm{g}}$

$0=-\frac{1}{2} \Omega_{\mathrm{k}} u_{r}-A \rho_{\mathrm{d}}\left(u_{\varphi}-w_{\varphi}\right)$

$0=2 \Omega_{\mathrm{k}} w_{\varphi}-A \rho_{\mathrm{g}}\left(w_{r}-u_{r}\right)$

$0=-\frac{1}{2} \Omega_{\mathrm{k}} w_{r}-A \rho_{\mathrm{g}}\left(w_{\varphi}-u_{\varphi}\right)$.

The quantities $u$ and $w$ denote the velocity of the gas and the dust in a Keplerian comoving frame, respectively. The subscripts $r$ and $\varphi$ indicate the radial and the azimuthal components of the velocities. The variables $\rho_{\mathrm{g}, \mathrm{d}}$ denote the mass densities of gas and dust and the quantity $A$ is defined as $A=\Omega_{\mathrm{k}} / \rho_{\mathrm{g}} \mathrm{St}$. We are primarily interested in the radial dust velocity of the Nakagawa-Sekiya-Hayashi solution (NSHs) which can be expressed as

$u^{\mathrm{NSH}}=\frac{2}{\psi \mathrm{St}+\frac{1}{\psi \mathrm{St}}} \psi v_{\mathrm{N}} \quad$ and $\quad \psi=\frac{1}{1+\epsilon}$.

The quantity $\epsilon \equiv \rho_{\mathrm{d}} / \rho_{\mathrm{g}}$ denotes the local dust-to-gas ratio. When $\psi \rightarrow 1$, i.e., when the dust-to-gas ratio is approaching zero, Eq. (7) reduces to the corresponding equation for single-particle drift, Eq. (3). The difference between these two equations is the additional factor $\psi$ that modifies the Stokes number St and the maximum drift velocity $v_{\mathrm{N}}$. Since $\psi$ is always smaller than one, the collective radial drift of the dust will always be smaller than the individual particle drift.

Taking collective effects into account requires knowledge about the dust density. Therefore, certain disk parameters, i.e. the turbulence parameter $\alpha$ as well as the initial dust-to-gas ratio $\epsilon_{0}$ before sedimentation, become of importance. Another dimensionless number, which now comes into play, is the turbulence parameter $q$. This number determines whether turbulent 


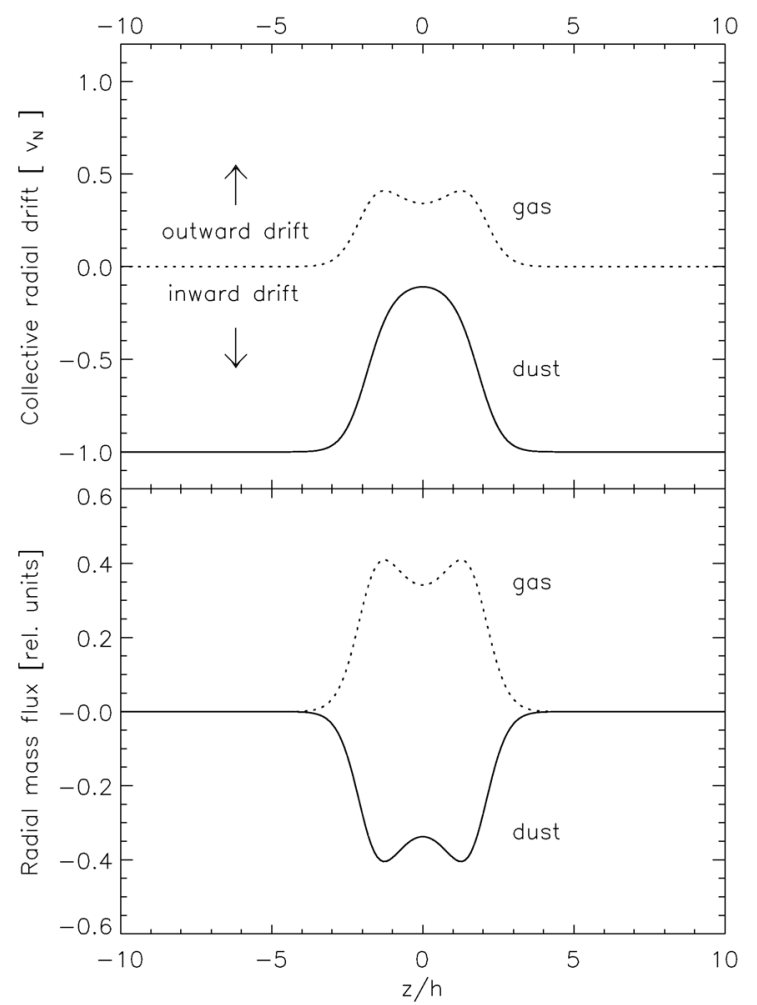

Fig. 5. The upper figure shows the collective radial velocities of gas and dust of the laminar NSH solution in terms of $v_{\mathrm{N}}$ as a function of height above the midplane. The lower figure shows the radial mass flux of gas and dust in arbitrary units. The values applied in this calculation are St $=1, \alpha=10^{-5}, q=1 / 2$ and $\epsilon_{0}=10^{-2}$.

diffusion is realised by small turbulent eddies moving fast or by big eddies moving slow (cf. Appendix A.1). These quantities determine the thickness of the dust layer $h$ and, hence, the dust density (cf. Eqs. (A.16) and (A.18)). In these equations we assume that the dust density in the vertical direction has a gaussian shape. This ansatz might be put into question if the turbulence is self-induced (Weidenschilling 1979). Although Johansen et al. (2006a) showed that the vertical dust density in self-induced turbulence has a gaussian shape for canonical dustto-gas ratios, the vertical structure can show a different shape especially when $\epsilon$ is increased for instance through photoevaporation (Weidenschilling 2006).

Since the dust density is a function of height above the midplane $z$, the radial drift velocities are dependent on $z$ as well. This vertical dependency is shown in Fig. 5 for an exemplary $\mathrm{NSH}$ solution. In this calculation we applied the values $\mathrm{St}=1$, $\alpha=10^{-5}, q=1 / 2$ and $\epsilon_{0}=10^{-2}$.

The plot shows that the dust moves inwards while the gas moves outwards which is generally the case in the NSH solution. In the higher regions of the disk $(|z|>3 h)$ the dust-to-gas ratio is much smaller than unity causing the collective drift behaviour to match the individual particle drift. However, closer to the midplane of the disk collective effects become important. With increasing dust-to-gas ratio towards the midplane, the radial inward drift of the dust decreases while the gas starts to move outwards. The clear difference in velocities between the collective drift and the individual drift around the midplane along with the fact that most of the dust is located in this region demonstrates the importance of collective effects for disks with low turbulence.

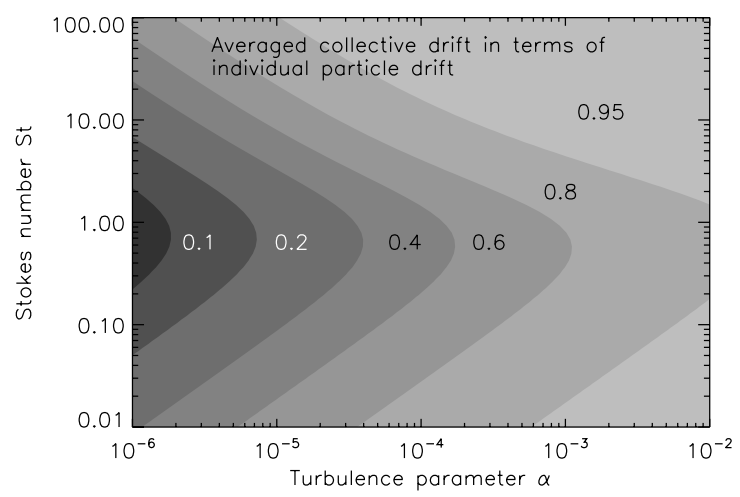

Fig. 6. A contour plot of the vertically averaged collective radial drift velocities of the dust in terms of the individual particle drift velocity as a function of turbulence parameter $\alpha$ and Stokes number St. The numbers in the diagram indicate the contour level and are related to the line on the left, respectively. The parameters for this calculation are $q=1 / 2$ and $\epsilon_{0}=10^{-2}$.

The radial velocities as a function of height above the midplane do not directly tell something about the entire radial flow of the dust since the dust itself is vertically distributed in a certain way. For this reason we will now calculate the vertically averaged radial velocity of the dust. This integrated velocity is given by

$\bar{u}^{\mathrm{NSH}}=\frac{1}{\Sigma_{\mathrm{d}}} \int_{z} \rho_{\mathrm{d}}(z) u^{\mathrm{NSH}}(z) \mathrm{d} z$.

A contour plot of this quantity as a function of the turbulence parameter $\alpha$ and the Stokes number St is shown in Fig. 6. The drift velocities in this diagram were expressed in terms of the corresponding individual particle drift velocity in order to explicitly point out the differences between these two models.

The figure shows that for fixed Stokes numbers the deviation increases continuously with lower turbulence in the disk. Lower $\alpha$ values imply thinner dust layers and, therefore, higher dust-togas ratios. With higher dust-to-gas ratios the back reaction of the dust to the gas increases, and hence the deviation between individual and collective drift velocities. One obvious solution to the whole radial drift problem of grains in the outer parts of the disk would be to continuously decrease the amount of turbulence in the disk or even to set $\alpha$ to zero. However, Weidenschilling (1979) has shown that a shear-instability between the dust layer and the gas induces a weak, but non-negligible level of turbulence. This turbulence is called "self-induced turbulence" which constrains the $\alpha$-value to be at least of the order of $\sim 10^{-6}$ (cf. Appendix B).

For fixed $\alpha$ Fig. 6 shows that for low Stokes numbers (small grains) the drift behavior approaches the individual particle drift. Low Stokes numbers imply thick dust layers, causing low dustto-gas ratios. For high Stokes numbers (large grains), very thin dust layers are obtained. One would intuitively think that this maximizes the collective effects. However, as can be seen from Eq. (7), in the limit of St $\rightarrow \infty$ one gets $u^{\mathrm{NSH}} \rightarrow 2 v_{\mathrm{N}} / \mathrm{St}$ which is equal to the individual particle drift of Eq. (3). So for large St the radial drift indeed drops, but not due to collective effects.

For a Stokes number of unity and a turbulence parameter of $10^{-6}$ the dust-to-gas ratio in the midplane is given by

$\epsilon_{\text {mid }}=\epsilon_{0} \frac{H}{h}=\epsilon_{0} \sqrt{\frac{\mathrm{St}}{\alpha}}=10$.

Therefore, the collective radial drift in the midplane in terms of the individual particle drift according to Eq. (7) is 


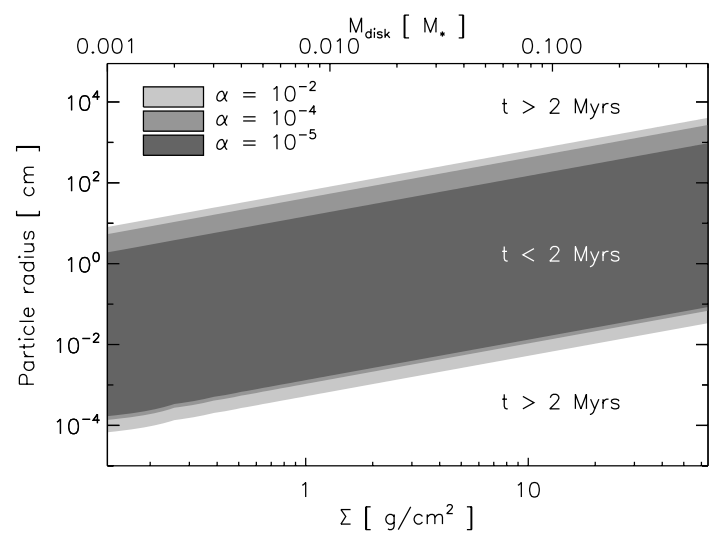

Fig. 7. As Fig. 4, but now with collective effects of the dust and the gas included, at $r=100 \mathrm{AU}$. Different grey scales are the results for different levels of turbulence, hence different thicknesses of the dust midplane layer. Note that for $\alpha=10^{-2}$ the results are virtually identical to the single-particle case (Fig. 4), since in this case the dust layer is so thick that the dust-to-gas ratio is much less than unity.

$u_{\text {mid }}^{\mathrm{NSH}} / v_{\mathrm{N}}=(1+\epsilon)^{-2}=0.008$. However, the vertically averaged drift velocity of the dust in terms of the individual particle drift in Fig. 6 is only 0.07, which is almost one order of magnitude higher. The reason for this is that the largest radial dust mass flux is not in the midplane, but slightly above the midplane (see Fig. 5). The mass flux is the product of dust density $\rho_{\mathrm{d}}$ and dust radial velocity $u^{\mathrm{NSH}}$. Although $\rho_{\mathrm{d}}$ drops strongly slightly above the midplane, the radial velocity $u^{\mathrm{NSH}}$ increases even faster, so that the product $\rho_{\mathrm{d}} u^{\mathrm{NSH}}$ has a maximum slightly above the midplane. While the formation of a dense dust layer can reduce the radial drift velocity in the midplane by a factor of 100 , the vertically averaged radial drift velocity can be only reduced by a factor of at most 10 .

\subsubsection{Radial drift times including collective effects}

Armed with the above drift velocity expressions we now calculate the conditions on particle radius and particle porosity that provide time scales larger than $2 \mathrm{Myr}$ taking into account collective effects.

At first we focus on the conditions on the particle radius. The interval of this particle property that corresponds to time scales shorter than $2 \mathrm{Myr}$ is shown in Fig. 7 for different $\alpha$-parameters. The second turbulence parameter $q$ is fixed at $1 / 2$ at all times, the initial dust-to-gas ratio is $10^{-2}$ and the filling factor $f$ is unity. All other parameters were already mentioned in the last section and are not changed throughout the paper unless directly stated.

According to this plot, the critical particle radii that provide the requested time scales hardly differ from the critical particle radii of the individual particle drift calculated in the last section. Even for small turbulence parameters which favour collective effects the time scales for $\mathrm{mm}$ to $\mathrm{cm}$ size particles are shorter than 2 Myr for any disk mass considered. The reason for this is that for very high and very low Stokes numbers, like the two critical numbers $\mathrm{St}_{-}$and $\mathrm{St}_{+}$representing the boundaries of the grey areas in Fig. 7, collective effects play a minor role (see Fig. 6, and discussion in the last subsection). The Stokes numbers for which the collective effects play the strongest role lie in the middle of these grey areas, i.e. where the drift time scales are anyway much too short to be compatible with the observations of $\mathrm{mm}$-sized particles in protoplanetary disks.

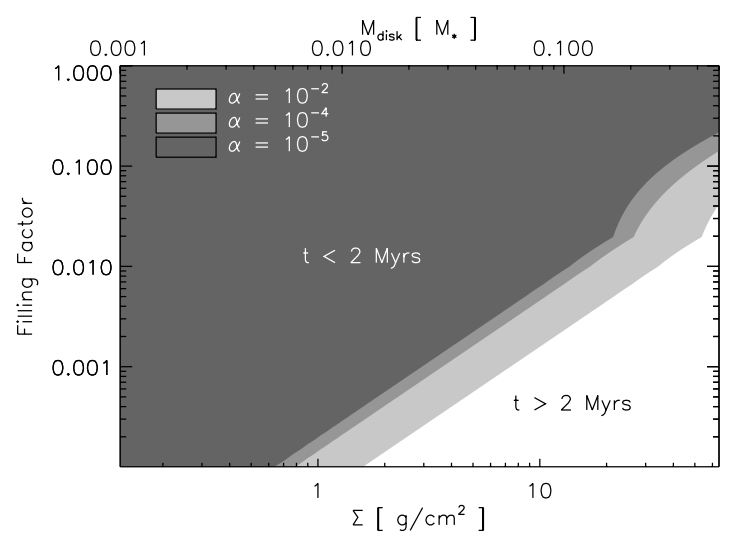

Fig. 8. The dust particle filling factor that provides time scales larger than 2 Myr as a function of disk mass for different turbulence parameters at $r=100 \mathrm{AU}$ in the disk. In this calculation collective effects of dust and gas are taken into account.

So what about the effects of fractal or porous growth? For simplicity we set the mass of the dust particle to be $1 \mathrm{~g}$ and then calculate the particle filling factor that provides time scales larger than 2 Myr. For a filling factor of unity a dust particle of $1 \mathrm{~g}$ corresponds to a particle radius of $1 / 2 \mathrm{~cm}$. For $f=10^{-4}$ the particle radius can be calculated to be $11 \mathrm{~cm}$. Like in the last paragraph we will perform the time scale calculation in dependency on the disk mass. The results of this calculation is shown in Fig. 8 for different values of the turbulence parameter $\alpha$. This diagram shows that for filling factors lower than $10^{-2}$ the time scale exceeds 2 Myr subject to the condition that the disk mass is higher than $\sim 0.2 M_{\star}$. For even higher disk masses the filling factor may exceed 0.1 for certain turbulent $\alpha$ parameters. This filling factor corresponds to a particle radius of $1 \mathrm{~cm}$. Ormel et al. (2007) showed that particle growth in protostellar disks can be associated with filling factors of less than $\sim 10^{-1}$. Therefore fractal growth seems to be an actual possibility to considerably increase the radial drift time scales of the dust. We will come back to that point in the discussion.

\subsection{Step 3 - Effect of turbulent viscosity}

We will now investigate the role of turbulent viscosity in addition to the effects studied so far. Including viscosity terms will have the opposite effect on the drift velocities than the collective effects. It will increase the radial drift of the dust and shorten the drift time scales. We will give the Navier-Stokes equation (NSe) including collective effects and viscosity terms and solve these equations numerically. However, we would like to discuss first why turbulent vicosity increases the radial drift of the dust. Under certain conditions, i.e. small turbulence parameters $\alpha$ or high Stokes numbers St, the previous sections have shown that the dust may settle into a thin midplane layer. When the dust-togas ratio inside this layer exceeds unity the gas is dragged along with the dust. Both components, dust and gas, tend to move with Keplerian velocity.

On the other hand, above the dust layer the dust-to-gas ratio is much smaller than unity. In this region the dust particles feel a continuous head wind which forces them to move with slightly sub-Keplerian velocity. This vertical decrease in azimuthal velocity from Keplerian velocity in the midplane to sub-Keplerian velocity in the higher regions of the disk produces a nonlinear velocity gradient in both the gas and the dust. 
If we include viscosity in our considerations it attempts to damp nonlinear spatial velocity differences. The vertical velocity gradient described above represents such a difference. Turbulent viscosity now acts in such a manner that it transports angular momentum from the midplane to the higher regions of the disk. While the midplane, the region where most of the dust is located, loses angular momentum and falls inward, the regions above the midplane gain angular momentum and move outward (cf. Fig. 1). This mechanism of vertical angular momentum exchange was first investigated by Youdin \& Chiang (2004).

\subsubsection{Navier-Stokes equations}

The Navier-Stokes equations for this problem are basically the set of Eqs. (6) plus some second order derivative terms due to the inclusion of viscosity

$$
\begin{aligned}
& 0=2 \Omega_{\mathrm{k}} u_{\varphi}-A \rho_{\mathrm{d}}\left(u_{r}-w_{r}\right)+v_{\mathrm{g}} \partial_{z}^{2} u_{r}-\frac{1}{\rho_{\mathrm{g}}} \partial_{r} p_{\mathrm{g}} \\
& 0=-\frac{1}{2} \Omega_{\mathrm{k}} u_{r}-A \rho_{\mathrm{d}}\left(u_{\varphi}-w_{\varphi}\right)+v_{\mathrm{g}} \partial_{z}^{2} u_{\varphi} \\
& 0=2 \Omega_{\mathrm{k}} w_{\varphi}-A \rho_{\mathrm{g}}\left(w_{r}-u_{r}\right)+\frac{v_{\mathrm{d}}}{\rho_{\mathrm{d}}} \partial_{z}\left(\rho_{\mathrm{d}} \partial_{z} w_{r}\right) \\
& 0=-\frac{1}{2} \Omega_{\mathrm{k}} w_{r}-A \rho_{\mathrm{g}}\left(w_{\varphi}-u_{\varphi}\right)+\frac{\nu_{\mathrm{d}}}{\rho_{\mathrm{d}}} \partial_{z}\left(\rho_{\mathrm{d}} \partial_{z} w_{\varphi}\right) .
\end{aligned}
$$

Hence, the algebraic Eqs. (6) turn into four coupled, differential equations of second order. The left hand side of the NavierStokes equations representing the time dependencies are set to be zero since we are interested in steady state solutions. The vectors $\boldsymbol{u}$ and $\boldsymbol{w}$ denote the velocities of the gas and the dust, respectively. The first terms on the right side correspond to the Coriolis force. These terms arise from the fact that the equations are formulated in a comoving frame. The second term represents the drag force coupling between the gas and the dust. The effects of viscosity show up in the third terms. The expressions for the viscosity of the gas and the dust can be found in Appendix A. In the following we will denote viscosity terms which involve derivatives of radial (azimuthal) velocities as "radial (azimuthal) viscosity terms". The very last term in the first line is an extra force acting on the gas which is caused by a radial pressure gradient. This term is responsible for the gas moving slower than the dust and causes the radial drift. The densities of gas and dust serve as input for the Navier-Stokes equations.

Takeuchi \& Lin (2002) also investigated the effect of gas viscosity on the drift of dust particles, but they neglected collective effects. This allowed to solve the equations analytically. The drift of the dust particles in their calculations was a superposition of two different effects: the individual dust particle velocity with respect to the gas and the velocity of the gas itself.

The former part of the dust particle drift was discussed in detail in Sect. 2.1. The second part of the dust particle drift investigated by Takeuchi \& Lin was due to the gas accretion process. This process of the gas is associated with a certain radial accretion velocity. Since the dust is to some extent coupled to the motions of the gas the dust is carried along with the accreting gas which leads to an extra source of radial particle drift.

In the beginning of this section we described that gas viscosity also increases the radial drift of the dust when collective effects come into play, i.e. when the dust settles into a thin midplane layer and starts to affect the motions of the gas. This process is different from the single particle considerations discussed by Takeuchi \& Lin since it is caused by collective effects and not by gas accretion. In the following, we will estimate the ratio of these two radial drift velocities.

The additional drift due to the accretion process may be estimated by a characteristic accretion velocity of the gas which is given by $v_{\mathrm{acc}} \propto \alpha c_{\mathrm{s}}^{2} / V_{\mathrm{k}}$ (Shakura \& Sunyaev 1973). Viscous collective effects imply drift velocities of order $v_{\text {coll }}=c_{\mathrm{s}}^{3} / V_{\mathrm{k}}^{2} \epsilon_{0} \mathrm{Re}^{\star}$ (Weidenschilling 2003). The ratio

$\xi=\frac{v_{\mathrm{acc}}}{v_{\mathrm{coll}}}=\alpha \epsilon_{0} \operatorname{Re}^{\star} \frac{V_{\mathrm{k}}}{c_{\mathrm{s}}}$

has values of at most $\approx 10^{-1}$ for $^{3} \alpha=10^{-2}, \epsilon_{0}=10^{-2}, \mathrm{Re}^{\star}=10^{2}$, $V_{\mathrm{k}}=3 \times 10^{5} \mathrm{~cm} \mathrm{~s}^{-1}$ and $c_{\mathrm{s}}=3 \times 10^{4} \mathrm{~cm} \mathrm{~s}^{-1}$. For smaller turbulence parameters which we will consider in this paper the influence of gas accretion will be even lower.

\subsubsection{Numerical results}

The parameters for the simulation are St $=1.2, \alpha=10^{-6}$, $q=1 / 2, \epsilon_{0}=10^{-2}$. The results are shown in Fig. 9. The two parameters $\alpha$ and St are chosen in a way that effects of viscosity become visible. These values imply a dust-to-gas ratio of 5 in the midplane of the disk and a half thickness of the dusty midplane layer which is $\approx 0.002 \mathrm{H}$. The turbulent motions of the gas have a speed of $0.005 v_{\mathrm{N}}$. The dotted lines in the Fig. 9 indicate the analytical solution of the laminar NSH equations $(v=0)$ which were discussed in the last section. The solid lines in Fig. 9 indicate the numerical solution including viscosity which differs significantly from the NSH solution.

Let us focus on the radial dust velocity since we are primarily interested in radial drift time scales. The radial flow of the dust is significantly affected by turbulent viscosity if $\alpha$ is smaller than $10^{-4}$. In this regime the effect is largest for Stokes numbers $\approx 5$. For $\alpha>10^{-4}$ the radial flow is approximately the flow predicted by Nakagawa et al. and viscosity seems to play a minor role. The radial dust velocities in the midplane with and without viscosity terms may differ by a factor of 5 for small $\alpha$ parameters and $\mathrm{St} \approx 5$.

The azimuthal dust and gas velocities as a function of height above the midplane vary in a complex manner. However, for Stokes numbers smaller than unity the situation with regard to the azimuthal velocities simplifies. In this regime these velocities do hardly differ from the expression given by Nakagawa et al. and viscosity seems to be negligible.

The radial outflow of the gas, which is shown in Fig. 9, is reduced if turbulent viscosity is included. This decrease may be up to a factor of 30 for small St and $\alpha$ parameters. For turbulence parameters higher than $10^{-4}$ the radial net outflow of the gas differs less than $10 \%$ from the outflow predicted by the NSH equations.

\subsubsection{Width of the azimuthal gas velocity layer}

The calculation of radial drift velocities in protostellar disks including collective effects and effects of viscosity are a challenging topic. Most equations can not be solved analytically and only numerical solutions provide information on the evolution of these disks. Therefore, disk model simplifications often come into play.

One simplification is that the dust sub-disk is assumed to be extremely thin and thought to behave, to some extent, like a solid disk. This approximation is called "plate drag approximation" (Goldreich \& Ward 1973). Under this condition, the gas

\footnotetext{
${ }^{3}$ Estimated values at $100 \mathrm{AU}$.
} 


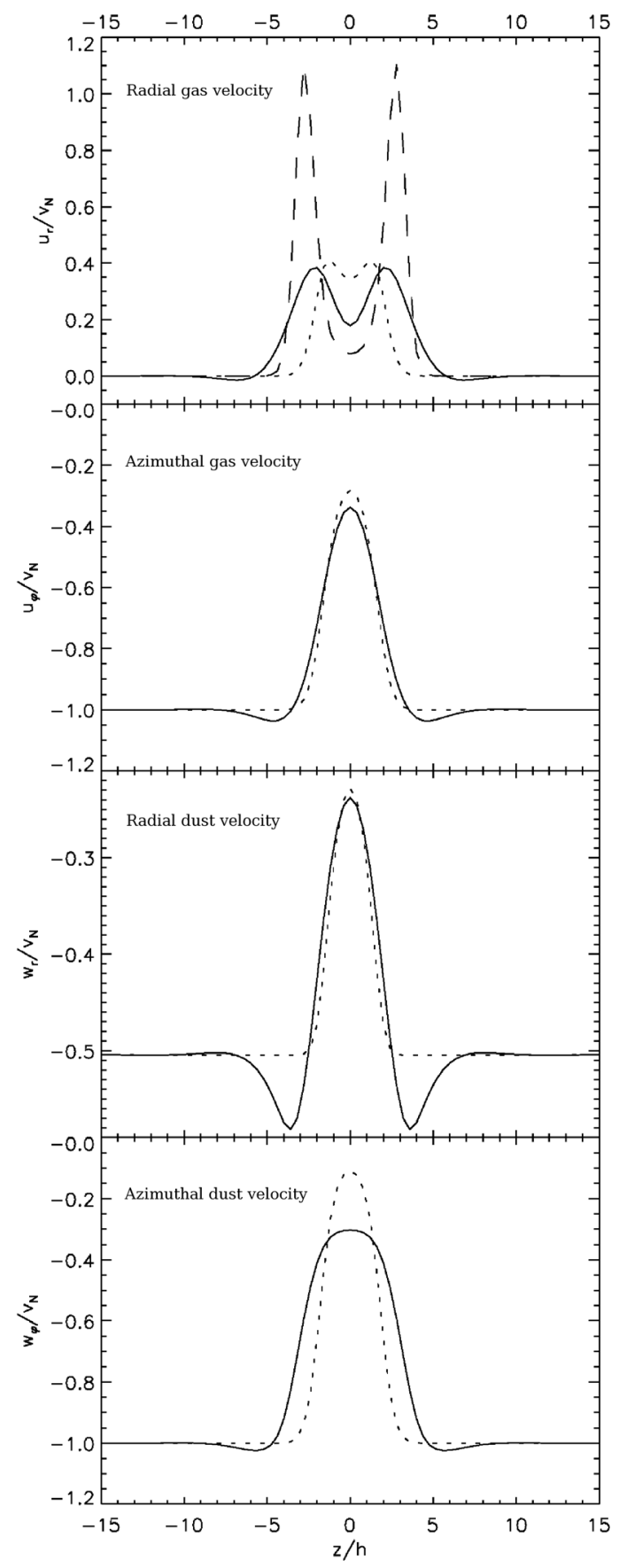

Fig. 9. The azimuthal and radial gas and dust velocities as a function of height above the midplane. The dotted lines denote the analytical normalised NSH solution without viscosity $(v=0)$ as discussed in Sect. 2.2. The solid lines indicate the numerical solution of the Navier-Stokes equations including azimuthal and radial viscosity terms. The dashed line in the top diagram shows the radial velocity of the gas if radial viscosity terms are neglected. The values for this simulation are $\mathrm{St}=1.2, \alpha=10^{-6}, q=0.5$ and $\epsilon_{0}=0.01$. Note that more than $80 \%$ of the dust in within the $z$-interval $[-2 h, 2 h]$.

layer above the dust layer can be described by an Ekman layer: the gas in the midplane is forced to move along with the Keplerian rotating solid equatorial subdisk. High above the midplane the gas is in equilibrium with the radial gas pressure gradient, yielding a slightly sub-Keplerian rotational velocity. The Ekman layer is the transitional region between these

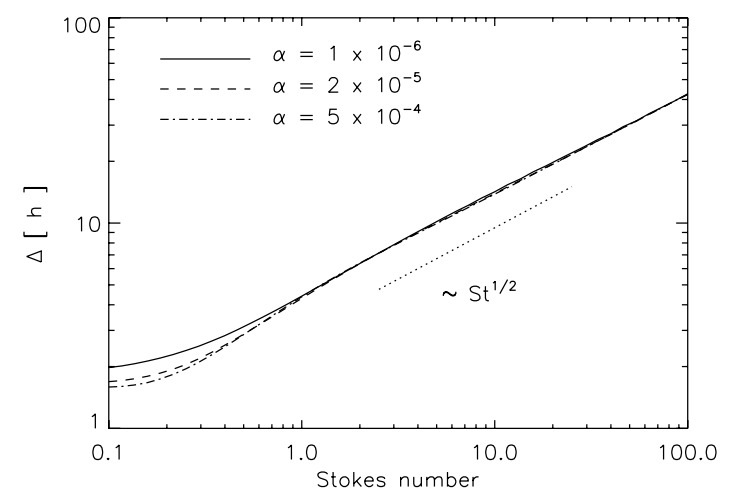

Fig. 10. The width of the azimuthal gas velocity distribution in units of the dust scale height $h$ as a function of Stokes number St for 3 different turbulence parameters $\alpha$. The dotted line indicates the $\sim \mathrm{St}^{1 / 2}$ dependency of the Ekman layer.

two extremes. The thickness of this layer depends on the viscosity of the gas.

In this subsection, we will compare our results with the predictions of the simplified model described above. We want to know the extent of the region where gas and dust affect each other and effects of viscosity become of importance. The comparison with regard to the drift velocities implied by this approximation, however, will be discussed in Sect. 2.3.6.

To quantify the length scale over which viscous collective effects play an important role, we define a measure $\Delta$ by

$\Delta(\alpha, \mathrm{St})=\int g(z)|z| \mathrm{d} z$.

The function $g(z)$ is given by

$g(z)=c_{N}\left|u_{\varphi}+v_{N}\right| z^{2}$.

The constant $c_{\mathrm{N}}$ provides the normalization of $g$. With this distribution function, deviations from the single particle solution $u_{\varphi}+v_{\mathrm{N}}$ are weighted in a way that differences high above the midplane are more important than differences close to the midplane. Therefore, $\Delta$ provides informations about the width of the vertical azimuthal velocity distribution of the gas. The dependence of this quantity as a function of St is shown in Fig. 10 for 3 different $\alpha$-values.

According to this diagram the value of the length scale $\Delta$ is a few dust scale heights as long as the Stokes number is smaller than unity. For higher St values this quantity increases exponentially up to more than $40 h$ for $\mathrm{St}=100$. We also find that $\Delta$ is hardly dependent on the turbulence parameter $\alpha$. This behaviour may be understood by investigating the length scale of the classical Ekman layer

$$
\frac{\Delta_{\mathrm{E}}}{h} \sim \frac{1}{h} \sqrt{\frac{\nu_{\mathrm{g}}}{\Omega_{\mathrm{k}}}} \sim \sqrt{\alpha} \frac{H}{h} \sim \sqrt{\mathrm{St}} .
$$

The dotted line in Fig. 10 indicates this dependency which shows that the gas layer in fact acts like an Ekman layer when the Stokes number exceeds unity.

\subsubsection{Vertical flow of angular momentum}

A remarkable effect of the viscosity is the radial inward drift of the gas which is impossible in the laminar NSH solution. At certain heights above the midplane the gas moves inwards (see Fig. 9 at $z= \pm 8 h$ for example). 


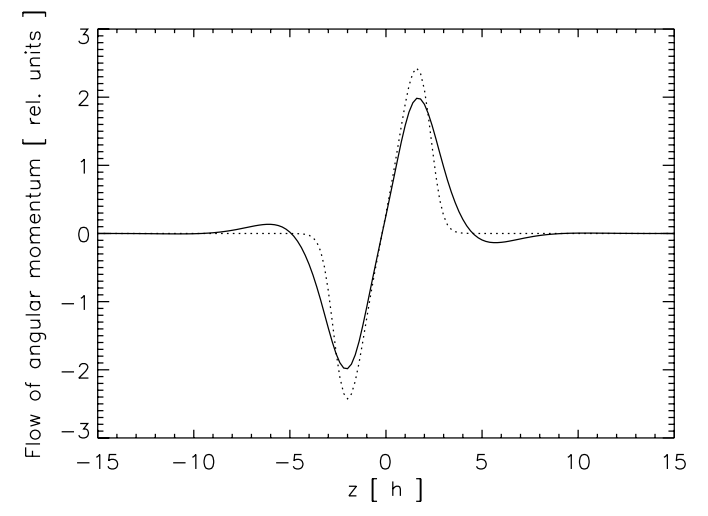

Fig. 11. This graph shows $\partial_{z} u_{\varphi}$ which indicates the flow of angular momentum in the vertical direction. The solid and the dotted lines show the flow of momentum with and without radial viscosity terms, respectively. The azimuthal viscosity terms are included at all times.

To understand this effect, we provide the basic scenario. The gas in the midplane of the disk dragged by the dust moves azimuthally faster than the gas outside the dust layer, which causes a vertical velocity gradient. Since viscosity tries to equalize such velocity gradients the gas in the higher regions of the disk is accelerated, decelerating the gas in the midplane. Therefore, viscosity transports angular momentum from the midplane to the higher regions of the disk.

To substantiate this effect we calculate the flow of angular momentum of the gas in the vertical direction. The structure of this flow can be analysed by calculating $\partial_{z} u_{\varphi}$ (see Fig. 11). This calculation was performed with the same parameter values as used in the last section.

The results show that the maximum vertical upward flow of angular momentum takes place at $\pm 2 h$. It also shows that there is a vertical downward flow of angular momentum at about $\pm 6 h$. This flow is strongly associated with the radial inward drift of the gas at certain heights of the disk and the inclusion of radial viscosity terms. This behaviour can be understood by performing simulations without radial viscosity terms:

If only azimuthal viscosity terms are included the azimuthal gas velocities continuously decrease with increasing distance from the midplane. This means that angular momentum is generally transported in the higher regions of the disk and never towards the midplane. This suggests that the vertical downward flow of angular momentum is an effect caused by radial viscosity. To substantiate this assumption Fig. 11 also shows the vertical flow of angular momentum when radial viscosity terms are neglected (dotted lines). The inflow vanishes in this case.

We also calculated the radial velocity of the gas without radial viscosity terms included. The results of this calculation indeed demonstrate that the radial inward drift of the gas vanishes in this case (see Fig. 9). The results also show the occurrence of two narrow peaks in the vertical velocity distribution of the radial gas velocity without radial viscosity terms. These peaks imply high velocity gradients. Radial viscosity, once included in the simulation, reduces these velocity differences by radially accelerating the neighbouring regions. This acceleration leads to a decrease in the azimuthal velocities since $\dot{u}_{\varphi} \sim-u_{r}$ due to Coriolis forces. This again causes the gas to drift inward.

Figure 9 also shows that the radial outflow of the gas may be faster than $v_{\mathrm{N}}$ if radial viscosity terms are neglected. The azimuthal velocity differences in gas and dust that initially cause any drift behaviour are of the same order of magnitude. Therefore, it appears unjustified to neglect radial viscosity terms

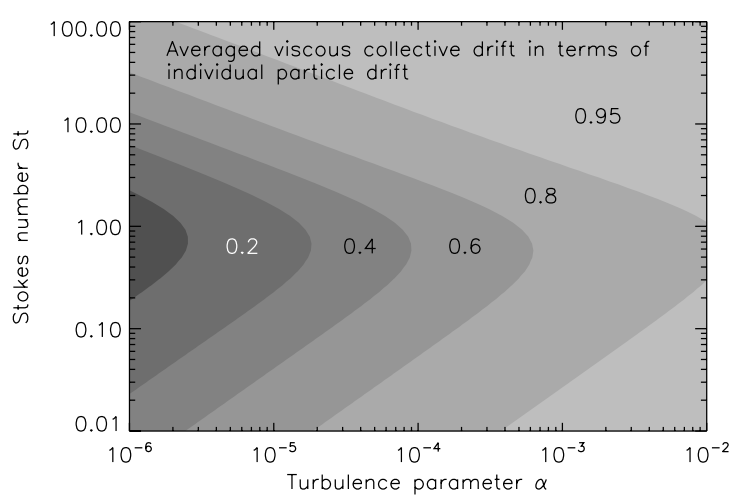

Fig. 12. A contour plot of the integrated radial dust velocity $\bar{u}^{\mathrm{V}}$ under the influence of viscosity as a function of turbulence parameter $\alpha$ and Stokes number St. The numbers related to the contour lines on the left side, respectively, indicate the net drift velocity in units the individual particle drift $u$.

as often implicitly done by using the plate drag approximation for example.

\subsubsection{Integrated radial velocities}

We now calculate the net flow of the dust $\bar{u}^{\mathrm{V}}$ according to Eq. (8). The result is shown in Fig. 12 expressed in terms of the individual particle drift velocity. According to these results the drift behaviour for high turbulence parameters is that of individual particles and neither collective effects nor effects of viscosity seem to play a major role in this part of the diagram. The net dust velocity has values of about $-v_{\mathrm{N}}$ for St $\approx 1 / 2$ (cf. Fig. 12) and decreases with lower $\alpha$ values and with growing distance from $\mathrm{St} \approx 1 / 2$.

To demonstrate how viscosity changes the collective drift behaviour investigated in step 2, it is suggestive to express the viscous collective drift $\bar{u}^{\mathrm{V}}$ in terms of the NSH drift $\bar{u}^{\mathrm{NSH}}$. A contour plot of this ratio can be seen in Fig. 13. This plot shows that the radial velocities calculated in this section exceed the drift due to collective effects by a factor of 2 at most if very low turbulence parameters are considered. For $\alpha$ parameters higher than $10^{-4}$ viscosity alters the drift scales by a factor of 1.2 in the most extreme case. The deviation from individual particle velocities due to collective effects were more pronounced than those due to viscosity. Therefore, we conclude that the drift behaviour is predominantly determined by collective effects and not by effects of viscosity.

\subsubsection{Plate drag approximation}

Here we will compare our results with previous work. We will consider the predictions of the "plate drag approximation". We would like to investigate if these two drift models predict the same radial velocities in certain parameter regimes.

In the plate drag approximation the drift induced by viscosity is given by (Goldreich \& Ward 1973; Weidenschilling 2003)

$u_{\mathrm{PD}}=\frac{\eta V_{\mathrm{k}}}{c_{\mathrm{S}} \epsilon_{0} \operatorname{Re}_{\star} \sqrt{2 \pi}}$.

The derivation of this expression is based on the assumption that the dust sublayer behaves like a solid disk subject to viscous stress on its surface by a turbulent boundary layer. This stress extracts angular momentum from the dust layer which implies radial drift of the dust (Weidenschilling 2006). The quantity $\mathrm{Re}_{\star}$ 


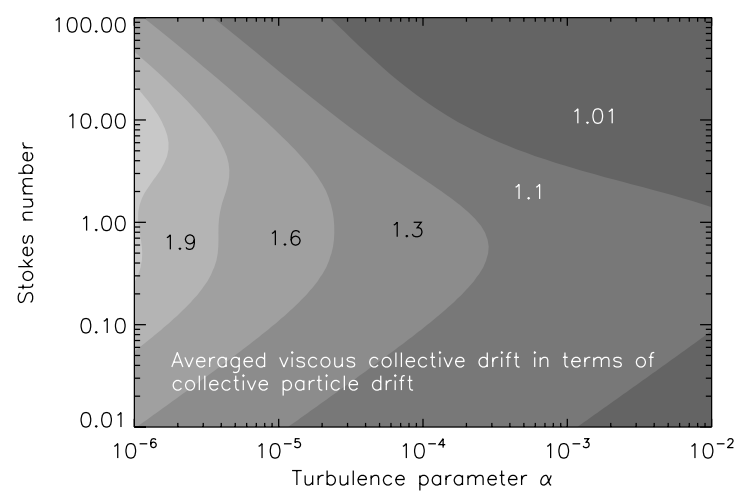

Fig. 13. A contour plot of the integrated radial dust velocity $\bar{u}^{\mathrm{V}}$ under the influence of viscosity as a function of turbulence parameter $\alpha$ and Stokes number St. The numbers related to the contour lines on the left side, respectively, indicate the net drift velocity in units of the NSH drift $\bar{u}^{\mathrm{NSH}}$ without viscosity.

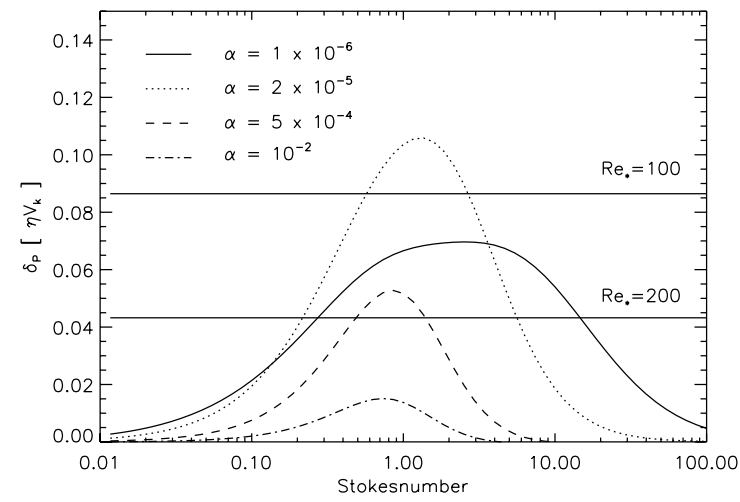

Fig. 14. The radial velocity deviation induced by the inclusion of viscosity as a function of Stokes number St for different turbulence parameters $\alpha$. The horizontal lines indicate the viscous radial drift predicted by the plate drag approximation for two different critical Reynolds numbers.

denotes the critical Reynolds number which indicates the transition point between laminar and turbulent flow. The value of this number depends on the geometry of the flow and is usually determined experimentally. We calculated the viscous drift for two different critical Reynolds numbers $\mathrm{Re}_{\star}=100$ and $\operatorname{Re}_{\star}=200$.

We will measure the radial drift due to the inclusion of viscosity by the deviation

$\delta_{\mathrm{p}}=\bar{u}^{\mathrm{V}}-\bar{u}^{\mathrm{NSH}}$.

This difference velocity $\delta_{\mathrm{p}}$ as well as the predictions of the plate drag approximation $u_{\mathrm{PD}}$ are shown in Fig. 14 as a function of the Stokes number for different turbulence parameters $\alpha$.

Figure 14 shows that the predictions of these two models are roughly of the same order of magnitude if the Stokes number is about unity. For Stokes numbers much smaller/larger than unity the results of the numerical integration of the Navier-Stokes equations deviates from the predictions of the plate drag model. Already Youdin \& Chiang (2004) put the plate drag approximation in question. They found that the plate drag overestimates turbulent stresses and that vertical shear and buoyancy are important elements missing in this description. While the plate drag model involves a radial drift velocity which is inversely proportional to the surface density of the layer and not explicitly dependent on particle size Weidenschilling (2006) found the very

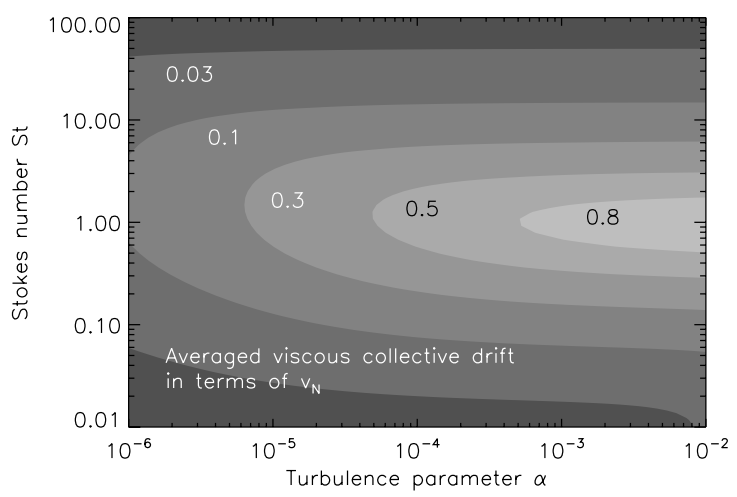

Fig. 15. A contour plot of the integrated radial dust velocity $\bar{u}^{\mathrm{V}}$ under the influence of viscosity as a function of turbulence parameter $\alpha$ and Stokes number St. The numbers related to the contour lines on the left side, respectively, indicate the net drift velocity in units of $-v_{\mathrm{N}}$.

contrary. In his simulations, the drift velocity shows no significant variation with surface density, but is dependent on particle size which clearly speaks against the validity of the plate drag model.

\subsubsection{Fitting formula}

A simple fitting formula that reproduces the results might be useful for forthcoming purposes for example investigations of drift time scales or radial mixing. For this reason we fitted the vertically averaged radial dust velocities given by the numerical solution of Eq. (10). This solution includes all effects investigated in this paper, i.e. collective effects and effects of turbulent viscosity. This result is shown in Fig. 15.

For the fit we use an expression of the form

$u_{\mathrm{fit}}=\frac{\Gamma(\alpha)}{x(\alpha, \mathrm{St})+\frac{1}{x(\alpha, \mathrm{St})}}$,

following Eq. (3) for individual particle drift. In this expression the amplitude $\Gamma$ is solely a function of the turbulence parameter $\alpha$. The quantity $\Gamma / 2$ matches the maximum occuring radial dust velocity in units of $-v_{\mathrm{N}}$ when $\alpha$ is fixed. The parameter $x$ is given by

$x(\alpha, \mathrm{St})=10^{\xi(\alpha)} \mathrm{St}^{\mu(\alpha)}$.

The two functions $\xi$ and $\mu$ are only dependent on $\alpha$. The parameter $\xi$ determines the location of the maximum of the velocity distribution, the parameter $\mu$ determines the width of the velocity distribution. The fits for the three functions $\Gamma, \xi$ and $\mu$ were performed with polynomials of the form

$\Gamma=\sum_{\mathrm{j}=0}^{4} c_{\mathrm{j}}^{\Gamma} y^{\mathrm{n}}, \quad \xi=\sum_{\mathrm{j}=0}^{2} c_{\mathrm{j}}^{\xi} y^{\mathrm{n}}, \quad \mu=\sum_{\mathrm{j}=0}^{4} c_{\mathrm{j}}^{\mu} y^{\mathrm{n}}$,

in which $y$ is given by $y=\log _{10} \alpha$. For the dependency of $\xi$ on $\alpha$ a second order polynomial turned out to be sufficient. For the quantities $\Gamma$ and $\mu$ a fourth order polynomial provided a satisfying fit to the simulation results. The coefficients for all these polynomials are listed in Table 1 . The deviation between the fitting function and the simulation within the parameter intervalls St $\in\left[10^{-2}, 10^{2}\right]$ and $\alpha \in\left[10^{-6}, 10^{-2}\right]$ is always smaller than $0.01 v_{\mathrm{N}}$. 
Table 1. Coefficients for the polynomical fit of the simulated integrated dust velocities.

\begin{tabular}{cccc}
\hline \hline$i$ & $c^{\Gamma}$ & $c^{\xi}$ & $c^{\mu}$ \\
\hline 0 & 1.89082 & $2.06164 \times 10^{-2}$ & 0.57083 \\
1 & 0.14763 & $4.69938 \times 10^{-3}$ & -0.41644 \\
2 & 0.20912 & $-4.05442 \times 10^{-3}$ & -0.12910 \\
3 & $8.25120 \times 10^{-2}$ & - & $-1.24036 \times 10^{-2}$ \\
4 & $7.38181 \times 10^{-3}$ & - & $-4.09782 \times 10^{-4}$ \\
\hline
\end{tabular}

\subsubsection{Radial drift times including effects of viscosity}

While collective effects reduce the radial drift of the dust the additional inclusion of viscosity into the disk model again increases it. For this reason, the time scales implied by collective effects and effects of viscosity represent an intermediate regime between the time scales of individual particles and the time scales implied by collective effects.

\section{Other possibilities to increase the drift timescale}

We have seen that even with the creation of very dense midplane layers for very low $\alpha$ the radial drift is too fast to explain the observed millimeter flux of these disks. We will now discuss other possible solutions to this problem. We will first consider the effect of the dust-to-gas ratio on the drift time scales. We will then investigate the importance of the turbulence parameter $q$ and consider the possibility of non-linear effects which could play an important role.

\subsection{Dust-to-gas ratio}

In this subsection we will investigate the influence of the dustto-gas ratio on the drift time scales. To increase this quantity we will remove a certain fraction of the gas from the disk. This removal has important implications for the drift time scales. When gas is removed from the disk then the dust particles are less affected by the motions of the gas. This leads to thinner dust layers and hence to higher dust-to-gas ratios. For this reason collective effects become of importance which reduces the radial drift velocities according to Eq. (7). In this paragraph, we will investigate how much gas we have to remove from the disk to provide time scales larger than 2 Myr.

As in the last sections we calculate the particle radius interval in which the drift time scale is shorter than $2 \mathrm{Myr}$. These calculations were performed for two different turbulence parameters $\alpha=10^{-5}$ and $\alpha=10^{-6}$. The results of these simulations are shown in Figs. 16 and 17. In these figures the disk mass and the surface density are the "original" mass and surface density of the disk before the assumed gas depletion that is invoked to alter the dust-to-gas ratio.

The drift behaviour of the dust particles hardly changes if only a small fraction of the gas is removed. Both figures show that the critical particle radius interval is little affected by removing $50 \%$ of the gas, cf. Fig. 7. However, for higher dust-to-gas ratios the critical interval decreases continuously. Considering the case $\alpha=10^{-6}$ we find that cm particles are able to stay in the outer parts of the disk for "original" disk masses $<0.05 M_{\star}$ and $>0.2 M_{\star}$ if only $5 \%$ of the gas is left. The critical interval disappears completely if the total vertical dust-to-gas ratio $\epsilon=\Sigma_{\mathrm{d}} / \Sigma_{\mathrm{g}}$ exceeds 0.40 . For higher turbulence parameters the critical radius interval decreases slower with higher dust-to-gas ratios. We find that for $\alpha=10^{-5}$ the interval disappears for a dust-to-gas

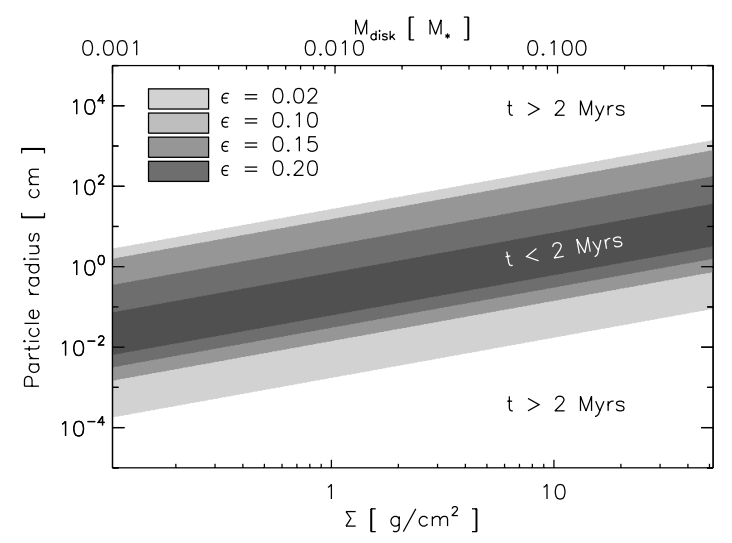

Fig. 16. This figure shows the effect of the dust-to-gas ratio on the drift time scales for a turbulence parameter of $\alpha=10^{-6}$. The figure gives the particle radius interval for which the drift time scale is smaller than $2 \mathrm{Myr}$ as a function of disk mass and surface density for different total vertical dust-to-gas ratios $\epsilon=\Sigma_{\mathrm{d}} / \Sigma_{\mathrm{g}}$. The surface density is given at 100 AU. The disk mass and the surface density in this figure are the "original" mass and surface density of the disk before the gas depletion that is invoked to alter the dust-to-gas ratio.

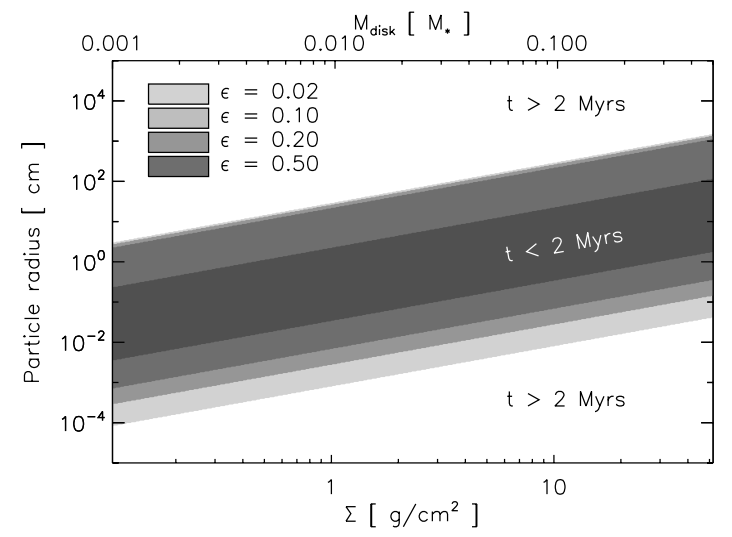

Fig. 17. This plot is the same as Fig. 16. But here the turbulent $\alpha$ parameter is $10^{-5}$.

ratio of $\epsilon=0.75$. We conclude that removing the gas may be a possibility to preserve $\mathrm{mm}$ to $\mathrm{cm}$ particles in the outer part of the disk.

\subsection{Turbulence parameter $q$}

Little attention was given to the turbulence parameter $q$ until now (cf. Appendix A.1). A certain diffusion coefficient of the gas may be realized by big gas eddies moving slow or by small gas eddies moving fast. These two extreme cases are represented by $q=1$ and $q=0$, respectively. To illustrate how strongly $q$ may influence the thickness of the dust layer we consider the following numerical example. We assume a Stokes number of unity and a turbulent $\alpha$ parameter of $10^{-3}$. For the extreme case $q=0$ we calculate a dust scale height of $h / H=10^{-3}$ and for $q=1$ we obtain $h / H=3 \times 10^{-2}$. These two dust scale heights differ by a factor of 30 which possibly influences the drift time scales.

We calculated the effect of the turbulence parameter $q$ on the drift velocity for two different turbulence parameters $\alpha$, i.e. $\alpha=10^{-6}$ and $\alpha=10^{-5}$. The Stokes number was chosen in a way the the drift velocity for $q=1 / 2$ corresponds to a timescale of 1 Myr. For $\alpha=10^{-6}$ and $\alpha=10^{-5}$ this implies St $=225$ 


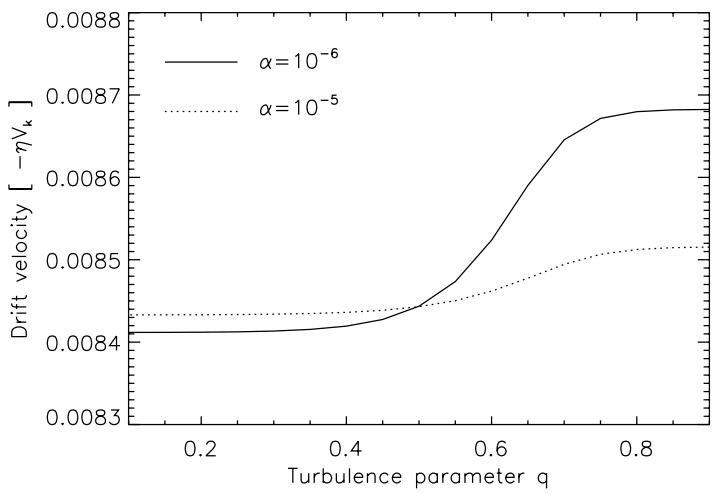

Fig. 18. This figure shows the effect of the turbulence parameter $q$ on the radial drift velocity for two different turbulence parameters $\alpha$. The Stokes number was chosen in a way that the radial drift velocity for $q=1 / 2$ corresponds to a timescale of $1 \mathrm{Myr}$ with regard to $100 \mathrm{AU}$ assuming that $v_{\mathrm{N}}=60 \mathrm{~m} / \mathrm{s}$. For $\alpha=10^{-6}$ and $\alpha=10^{-5}$ this implies $\mathrm{St}=225$ and $\mathrm{St}=235$, respectively.

and $\mathrm{St}=235$, respectively. The results of these simulations are shown in Fig. 18.

This figure shows that the integrated drift velocities of the dust vary by $3 \%$ for $\alpha=10^{-6}$ and by $1 \%$ for $\alpha=10^{-5}$ when $q$ is changed from zero to unity. We find that for higher $\alpha$ parameters this variation is always less than $0.4 \%$. We conclude that $q$ has a very minor effect on the radial drift velocities.

A small $h / H$ ratio leads to a high dust-to-gas ratio in the midplane of the disk. This causes the radial drift of the dust in the midplane to decrease due to collective effects. One would now intuitively think that a continuously decreasing $h / H$ ratio leads to smaller and smaller radial drift velocities but this is not the case for the following reason: When the ratio $h / H$ decreases the vertical gradients of the azimuthal gas and dust velocities increase. Therefore, more angular momentum is transported in the higher regions of the disk. The midplane loses angular momentum which directly causes the radial velocity of the dust to increase.

Finally both effects, the decrease in radial velocity due to collective effects and the increase of velocity due to the vertical transport of angular momentum, seem to cancel each other (or at most result in a negligible change in radial velocity) when small $h / H$ ratios are considered.

\subsection{Effects of non-linear dynamics}

So far we have considered different equilibrium states that would potentially allow solid particles to reside at large orbital radii for a longer time than a single test particle. It is however also possible for dynamical effects, such as spiral arms, turbulence or vortices, to reduce the radial drift.

Dust particles are forced to climb up the local gas pressure gradient. In the simple case of a gas pressure that falls monotonically with radius the particles fall into the inner disc, but the particles may end up in any local gas overdensity that they encounter on their way (Klahr \& Lin 2001; Haghighipour \& Boss 2003). If the disc is massive enough to be gravitationally unstable, its spiral arms may act as such local density maxima (Rice et al. 2004). Transient overdensities that occur in magnetorotational turbulence, in a way very elongated vortices, have the same effect (Johansen et al. 2006b), slowing down radial drift by a factor of two. The important parameter for reducing the overall radial drift is the life-time of the gas overdensity. Spiral arms would from this perspective be a good candidate, since turbulent overdensities tend to live no longer than a few local orbits of the disc.

The coupled flow of gas and dust is in itself unstable to the streaming instability (Youdin \& Goodman 2005), leading to particle clumping in the non-linear state (Johansen et al. 2006b). These local dust overdensities drag the gas along with their orbital motion, thus reducing the sub-Keplerian head wind and the radial drift. The effect of the streaming instability on the radial drift can be as high as a factor two in reduction (Johansen \& Youdin 2007).

\subsection{Temperature and surface density profiles}

We also investigated to which extent the radial drift time scales of the dust particles depend on the temperature and the surface density profile. These quantities were found to play a minor role which can be reasoned as follows. If the temperature is decreased by a factor of 2 then the maximum radial drift velocity $v_{\mathrm{N}}$ decreases by the same factor (cf. Eq. (A.22)). This means that if the temperature at $100 \mathrm{AU}$ is decreased from $20 \mathrm{~K}$ to a rather low value of $10 \mathrm{~K}$ then the drift time scales would only increase by a factor of 2 . Since the radial drift problem spans at least one order of magnitude a change in temperature does not provide a solution. A change in the surface density index from $\delta=0.8$ towards $\delta=0$ changes the maximum drift velocity $v_{\mathrm{N}}$ by a factor of 1.5 according to Eq. (A.22) which also does not solve the problem.

\section{Summary}

We discussed two different subjects. First, we investigated the velocity structure of the dust layer and the gas layer. We calculated typical radial drift velocities of the dust including several effects, i.e. collective effects and effects due to turbulent viscosity. The results of this first issue enabled us to estimate characteristic drift time scales of the dust which represents the second area of interest. These calculations were performed in order to explain interferometric millimeter observations that indicate the presence of $\mathrm{mm}$ to $\mathrm{cm}$ size particles in the outer part of disks with ages up to $10 \mathrm{Myr}$. In the following we will briefly summarize the results.

\subsection{Velocity structure of gas and dust layer}

First we discussed the individual particle drift of the dust (Whipple 1972; Weidenschilling 1977). In this model the dust particles are considered to be independent and the gas is assumed not to be affected by the dust at all. This scenario involves no vertical structure of the dust since only individual dust particles are considered.

We discussed the influence of collective behaviour on the radial drift velocities of the dust. Collective effects take place when the dust-to-gas ratio exceeds unity, e.g. when the dust sediments into a thin midplane layer due to low turbulence. By assuming an initial dust-to-gas ratio of 0.01 we found that the local radial drift velocity of the dust in the midplane of the disk may be reduced by a factor of 100 for small turbulence parameters and certain particle characterictics. The vertically averaged radial dust velocity is at least an order of magnitude lower than the corresponding individual particle drift.

The additional inclusion of turbulent viscosity in our considerations increased the radial drift of the dust due to the vertical 
transport of angular momentum. This increase in radial velocity was not as significant as the decrease by including collective effects. Turbulent viscosity enhanced the radial drift by a factor of 2 at most. Therefore, we conclude that collective effects play the major role in the parameter regimes considered in this paper.

It turned out to be neccessary to include radial viscosity terms of gas and dust. If these terms are neglected, the numerical solutions showed very steep and unrealistic velocity gradients. Radial viscosity, once included in the considerations, reduced these velocity differences which considerably changed the radial velocities of gas and dust.

For turbulence parameters $\alpha$ larger than $10^{-4}$ collective effects as well as effects of turbulent viscosity play a minor role. The dust is stirred up away from the midplane in such a way that the dust-to-gas ratio is too low for these effects to play a role. The radial drift velocities of the dust in this case were reduced by a factor of 1.3 at most. For this reason, both effects can be disregarded in this regime.

We compared the results of our numerical solutions to the predictions of the so-called "plate drag approximation". It was shown that both models roughly agree if the Stokes number of the particle is about unity. For Stokes numbers higher (lower) than unity the two models predict different radial velocities. Previous work already put the "plate drag approximation" into question (Youdin \& Chiang 2004) with similar conclusions.

Apart from radial drift behaviour some structural aspects of the gas layer and dust layer were also investigated. We calculated the extent of the vertical region in which gas and dust affect each other. This calculation showed that the gas layer behaves like a classical Ekman layer if Stokes numbers larger than unity are considered. For well coupled particles, i.e. for particles with Stokes number smaller than unity, the two descriptions differ significantly.

\subsection{Radial drift time scales}

We investigated the radial drift of dust particles with respect to collective behaviour and effects of viscosity under standart conditions, i.e. an initial dust-to-gas ratio of $10^{-2}$, standard turbulence parameter $q$, etc. We found that a possible solution for the drift problem are very high/low disk masses. Moreover, high porosity particles $(f<0.1)$ in low turbulent disks with masses $>0.2 M_{\star}$ provided drift time scales $>2 \mathrm{Myr}$.

However, except for these two cases the drift time scales of the dust turned out to be far too short to explain the millimeter observations. For this reason we investigated several possibilities in order to increase the drift time scales. We found that removing the gas from the disk may increase the time scales up to more than 2 Myr. For example, for a turbulence parameter of $\alpha=10^{-6}$ we found that $\mathrm{cm}$ size particles remain in the outer parts of the disk for more than $2 \mathrm{Myr}$ if disk masses $<0.05 M_{\star}$ or $>0.2 M_{\star}$ are considered and only $5 \%$ of the gas is left. If the disk is more turbulent more gas has to be removed in order to keep the particles in the outer parts for longer periods of time.

Gas might be removed in the later stages of disk evolution when photoevaporation sets in. While the gas is evaporated from the disk the dust particles $>20 \mu \mathrm{m}$ (Takeuchi \& Lin 2005; Bally et al. 2005) remain in the outer parts of the disk. However, current photoevaporation models remove the gas from the disk rather abruptly (Alexander et al. 2006). This would lead to rather high relative particle velocities in the disk. Hence, the dust particles would fragment and destructive collisions would play an important role. The centimeter particles would be destroyed in short time scales which is not in agreement with the observations.

We also investigated the effect of the turbulence parameter $q$ on the drift time scales and we found that this parameter plays a minor role. Varying this parameter between zero and unity, the most extreme cases possible, changed the drift velocities by $3 \%$ at most.

We did not consider the growth of the particles in this paper. The growth time scale of the dust to reach a centimeter in size could be of the order of 1 Myr considering the outer parts of the disk. A model including both processes, radial drift and coagulation, would clarify this issue. Recent work about the drift time scales in comparison to the growth time scales was done by Klahr \& Bodenheimer (2006). According to their calculations, the dust would long have drifted away from 100 AU before the particles even reach the size of about a centimeter.

Moreover, the effect of fragmentation could change the situation. Even though a process that destroys particles is included it could finally speed up the process of coagulation: fragmentation leads to a permanent amount of small particles as a result of collisions. These small particles may be swept up by larger particles due to their high relative velocity. Although some particles are destroyed, the final sum of both effects might lead to an accelerated growth. These effects, the radial drift, the dust particle coagulation and the effect of fragmentation, will be the topic of a forthcoming paper.

\subsection{Observational implications}

We have shown that thin midplane layers in low turbulent disks can conceivably explain the presence of the observed large amounts of $\mathrm{mm} / \mathrm{cm}$ sized grains if a significant fraction of the gas is removed, for instance through photoevaporation of the gas. Therefore, we should also discuss whether the presence of such thin midplane layers is consistent with observations of e.g. edge-on disks.

The infrared emission from protoplanetary disks originates from smaller ( $\ll 3 \mathrm{~mm}$ ) dust grains. These grains must be smaller than 3 micrometer, as can be inferred from the presence of a 10 micron silicate feature in emission in most sources. Even with relatively little vertical mixing (low turbulence) the very smallest dust particles can still be mixed up to intermediate height above the midplane (Dullemond \& Dominik 2004a), although we admit that the disk should look significantly less flared in such a case, i.e. the disk should be of Group II in the Meeus et al. (2001) classification. Interestingly, Acke et al. (2004) have shown that there is indeed a correlation between the presence of large grains in the outer regions of disks around Herbig stars and the type of SED of the disk: disks with large amounts of large $(\mathrm{mm} / \mathrm{cm})$ grains appear on average to also have SEDs consistent with flatter disk geometry. This seems to substantiate at least qualitatively the idea of low turbulent disks.

For larger grains, which can be observed at $\mathrm{mm} / \mathrm{cm}$ wavelengths, there have not been observations of edge-on disk with very thin mm disks so far. However, this has two reasons. The first reason is that if the $\mathrm{mm} / \mathrm{cm}$ disk is very thin, then the chance to observe it sufficiently precisely edge-on is very small. This reduces the number of potential candidates for such measurements drastically. The second reason is that current stateof-the-art interferometers do not yet have the spatial resolution to resolve such thin disks. For instance, the Butterfly Nebula (a well-studied nearly perfectly edge-on disk), was resolved with OVRO by Wolf et al. (2003), but the vertical extent of the 
observed disk was as large as the beam size, i.e. unresolved in vertical direction.

\section{Conclusion}

We find that the radial drift time scales for millimeter-sized grains observed in the outer $(\sim 100 \mathrm{AU})$ regions of protoplanetary disks are of the order of $10^{5}$ years, whereas the age of these disks is generally between $10^{6}$ and $10^{7}$ years. So, according to theory, the particles that are observed should have long ago drifted into the star, which is evidently inconsistent with observations. We have attempted to resolve this mystery by investigating what happens in the case of very low-turbulence disks in which the dust gathers in a thin midplane layer. The hope was that collective effects of the dust (by coupling to the gas) could slow down the drift. We find that this reduction factor is sufficient if either very high/low disk masses are considered or the particles have high porosities. In any other case this reduction factor is by far insufficient to resolve the problem. Moreover, we find that by removing a substantial fraction of the gas from the disk the drift time scales can be sufficiently enhanced. Another possibility is particle trapping in local gas pressure maxima (Klahr \& Henning 1997), but this topic was beyond the semianalytical model of this paper.

Acknowledgements. We wish to thank Carsten Dominik, Dmitry Semenov, Jens Rodmann and Karim Fertikh for useful discussions and comments. We also wish to thank the referee, Stu Weidenschilling, for useful criticism that helped us improve the paper.

\section{References}

Acke, B., van den Ancker, M. E., Dullemond, C. P., van Boekel, R., \& Waters, L. B. F. M. 2004, A\&A, 422, 621

Alexander, R. D., \& Armitage, P. J. 2007, MNRAS, 375, 500

Alexander, R. D., Clarke, C. J., \& Pringle, J. E. 2006, MNRAS, 369, 229

Balbus, S. A., \& Hawley, J. F. 1991, ApJ, 376, 214

Balbus, S. A., \& Hawley, J. F. 1998, Rev. Mod. Phys., 70, 1

Bally, J., Moeckel, N., \& Throop, H. 2005, in Chondrites and the Protoplanetary Disk, ed. A. N. Krot, E. R. D. Scott, \& B. Reipurth, ASP Conf. Ser., 341, 81 Barge, P., \& Sommeria, J. 1995, A\&A, 295, L1

Beckwith, S. V. W., Henning, T., \& Nakagawa, Y. 2000, Protostars and Planets IV, 533

Blum, J., \& Wurm, G. 2000, Icarus, 143, 138

Brandenburg, A., Nordlund, A., Stein, R. F., \& Torkelsson, U. 1995, ApJ, 446, 741

Chandrasekhar, S. 1961, Hydrodynamic and hydromagnetic stability, International Series of Monographs on Physics (Oxford: Clarendon)

Cuzzi, J. N., \& Weidenschilling, S. J. 2006, Particle-Gas Dynamics and Primary Accretion, Meteorites and the Early Solar System II, 353

Cuzzi, J. N., Dobrovolskis, A. R., \& Champney, J. M. 1993, Icarus, 106, 102
Dominik, C., Blum, J., Cuzzi, J. N., \& Wurm, G. 2007, in Protostars and Planets V, ed. B. Reipurth, D. Jewitt, \& K. Keil, 783

Dubrulle, B., Morfill, G., \& Sterzik, M. 1995, Icarus, 114, 237

Dullemond, C. P., \& Dominik, C. 2004a, A\&A, 417, 159

Dullemond, C. P., \& Dominik, C. 2004b, A\&A, 421, 1075

Fromang, S., \& Nelson, R. P. 2005, MNRAS, 364, L81

Garaud, P., Barrière-Fouchet, L., \& Lin, D. N. C. 2004, ApJ, 603, 292

Goldreich, P., \& Ward, W. R. 1973, ApJ, 183, 1051

Gómez, G. C., \& Ostriker, E. C. 2005, ApJ, 630, 1093

Haghighipour, N., \& Boss, A. P. 2003, ApJ, 583, 996

Hartmann, L., Calvet, N., Gullbring, E., \& D’Alessio, P. 1998, ApJ, 495, 385

Johansen, A., \& Klahr, H. 2005, ApJ, 634, 1353

Johansen, A., Henning, T., \& Klahr, H. 2006a, ApJ, 643, 1219

Johansen, A., Klahr, H., \& Henning, T. 2006b, ApJ, 636, 1121

Johansen, A., \& Youdin, A. 2007, ArXiv Astrophysics e-prints

Kempf, S., Blum, J., \& Wurm, G. 2000, BAAS, 32, 1099

Kitamura, Y., Momose, M., Yokogawa, S., et al. 2002, ApJ, 581, 357

Klahr, H., \& Bodenheimer, P. 2006, ApJ, 639, 432

Klahr, H. H., \& Henning, T. 1997, Icarus, 128, 213

Klahr, H. H., \& Lin, D. N. C. 2001, ApJ, 554, 1095

Launder, B. E. 1976, Heat and mass transport, Turbulence (A77-20355 07-34)

(Berlin and New York: Springer-Verlag), 231

McComb, W. D. 1990, Chemical Physics

Meeus, G., Waters, L. B. F. M., Bouwman, J., et al. 2001, A\&A, 365, 476

Nakagawa, Y., Sekiya, M., \& Hayashi, C. 1986, Icarus, 67, 375

Natta, A. 2004, in Debris Disks and the Formation of Planets, ed. L. Caroff, L. J. Moon, D. Backman, \& E. Praton, ASP Conf. Ser., 324, 20

Natta, A., Testi, L., Neri, R., Shepherd, D. S., \& Wilner, D. J. 2004, A\&A, 416, 179

Natta, A., Testi, L., Calvet, N., et al. 2007, in Protostars and Planets V, ed. B. Reipurth, D. Jewitt, \& K. Keil, 767

Ormel, C. W., Spaans, M., \& Tielens, A. G. G. M. 2007, A\&A, 461, 215

Rice, W. K. M., Lodato, G., Pringle, J. E., Armitage, P. J., \& Bonnell, I. A. 2004, MNRAS, 355, 543

Rodmann, J. 2006, Ph.D. Thesis, Combined Faculties for the Natural Sciences and for Mathematics of the University of Heidelberg, Germany. XIII+137 pp.

Rodmann, J., Henning, T., Chandler, C. J., Mundy, L. G., \& Wilner, D. J. 2006, A\&A, 446, 211

Schräpler, R., \& Henning, T. 2004, ApJ, 614, 960

Sekiya, M. 1998, Icarus, 133, 298

Shakura, N. I., \& Sunyaev, R. A. 1973, A\&A, 24, 337

Suttner, G., \& Yorke, H. W. 2001, ApJ, 551, 461

Takeuchi, T., \& Lin, D. N. C. 2002, ApJ, 581, 1344

Takeuchi, T., \& Lin, D. N. C. 2005, ApJ, 623, 482

Testi, L., Natta, A., Shepherd, D. S., \& Wilner, D. J. 2003, A\&A, 403, 323

Völk, H. J., Morfill, G. E., Roeser, S., \& Jones, F. C. 1980, A\&A, 85, 316

Weidenschilling, S. J. 1977, MNRAS, 180, 57

Weidenschilling, S. J. 1979, BAAS, 11, 552

Weidenschilling, S. J. 1980, Icarus, 44, 172

Weidenschilling, S. J. 2003, Icarus, 165, 438

Weidenschilling, S. J. 2006, Icarus, 181, 572

Whipple, F. L. 1972, in From Plasma to Planet, ed. A. Elvius, 211

Wilner, D. J., D’Alessio, P., Calvet, N., Claussen, M. J., \& Hartmann, L. 2005, ApJ, 626, L109

Wolf, S., Padgett, D. L., \& Stapelfeldt, K. R. 2003, ApJ, 588, 373

Youdin, A. N., \& Chiang, E. I. 2004, ApJ, 601, 1109

Youdin, A. N., \& Goodman, J. 2005, ApJ, 620, 459

Youdin, A. N., \& Shu, F. H. 2002, ApJ, 580, 494 
F. Brauer et al.: Radial drift of mm-cm size grains, Online Material $p 1$

\section{Online Material}




\section{Appendix A: Theoretical background}

The main ingredients of the models are the stellar parameters, the structure and mass of the disk, the description of the turbulence, and the description of the interaction between the dust and the gas. In principle, for the radial drift velocity we do not need knowledge of the turbulence (except for the turbulence effects described by Johansen et al. (2006b) which might reduce the drift by a factor of up to 2). However, turbulence sets the thickness of the dust midplane layer, and hence the density of the dust, which affects the drift velocity once this density comes close to, or exceeds the gas density. If there would be no turbulence at all, the dust particles would settle to the midplane and form a razor-thin layer qualitatively like the rings of Saturn. The more turbulence there is the harder it is for the dust to form a thin layer since it is mixed up and again transported in the higher regions of the disk. Turbulence is not yet understood in detail and there is various literature about this challenging topic (Völk et al. 1980; Schräpler \& Henning 2004; Balbus \& Hawley 1991, 1998). In this paper we will make use of the $\alpha$-prescription (Shakura \& Sunyaev 1973). This specific way of regarding turbulence is somewhat superficial since it ignores most details of turbulence. However, the advantage is that it makes turbulence manageable without extensive hydrodynamical simulations.

\section{A.1. $\alpha$-prescription for turbulent gas}

In this section we will give a short overview about our $\alpha$ prescription of particles in a turbulent disk. Turbulence mixes things up and therefore acts like a kind of diffusion. The diffusion coefficient ${ }^{4} D$ can be written as a product of a velocity scale $V_{0}$ and a length scale $L_{0}$,

$D=V_{0} \times L_{0}$.

A typical velocity scale is the isothermal soundspeed $c_{\mathrm{s}}^{2} \equiv$ $k T_{\text {gas }} / \mu$, with $T_{\text {gas }}$ the gas temperature, $k$ the Boltzmann constant, $\mu$ the mean molecular weight of the gas (which we take 2.3 times the proton mass for a standard mixture of molecular hydrogen and atomic helium). A characteristic length scale is given by the pressure scale height of the gas $H=c_{\mathrm{s}} / \Omega_{k}$, where $\Omega_{k} \equiv \sqrt{G M_{*} / r^{3}}$. Regarding these typical scales at a certain radius we can alternatively express the diffusion coefficient for the gas as follows

$D=\alpha c_{\mathrm{s}} H$.

The value of the parameter $\alpha$ reflects the amount of turbulence in the disk and it ranges from $10^{-6}$ (Weidenschilling 1980) up to $10^{-2}$ (Hartmann et al. 1998). Extensive hydrodynamical simulations show typical $\alpha$ values of about $10^{-3}$ (Brandenburg et al. 1995; Johansen \& Klahr 2005). In general we have to distinguish between the $\alpha$ introduced to determine the vertical distribution of the dust and the $\alpha_{\text {acc }}$ linked with the accretion process. Both quantities are related by $\alpha_{\text {acc }}=\operatorname{Pt} \alpha$ where Pt denotes the Schmidt number. We will assume $\mathrm{Pt}=1$ in this paper (Launder 1976; McComb 1990; Johansen \& Klahr 2005).

There is an ambiguity with regard to this formulation till now since $\alpha$ does not provide any information about $V_{0}$ and $L_{0}$. This can be seen in a better way by introducing a turbulence parameter $q$

$D=\alpha c_{\mathrm{s}} H=\alpha^{q} c_{\mathrm{s}} \times \alpha^{1-q} H$,

\footnotetext{
${ }^{4}$ All symbols used in this paper are listed in a table at the very end.
}

which ranges between 0 and 1 . Now we can identify the terms of Eq. (A.1) by defining $V_{0}=\alpha^{q} c_{\mathrm{s}}$ and $L_{0}=\alpha^{1-q} H$. The physical interpretation of $q$ is as follows. A certain diffusion can be realized by big eddies which move very slow $(q=1)$ or by small eddies moving very fast $(q=0)$. Both possibilities result in the same diffusion-coefficient. Normally $q$ is set to be $1 / 2$ (e.g. Schräpler \& Henning 2004) but there are other values possible. For example, in self-induced turbulence $q$ tends to be smaller than $1 / 2$ (Weidenschilling 2006). Another possibility are big convective cells of scale $H$ (Klahr \& Henning 1997) which would imply $q=1$. The fundamental importance of knowledge about $q$ can be understood by calculating the turn-over-eddytime $\tau_{\text {ed }}$

$\tau_{\mathrm{ed}}=\frac{L_{0}}{V_{0}}=\frac{2 \pi}{\omega_{\mathrm{ed}}}=\alpha^{1-2 q} \frac{1}{\Omega_{k}}$

where $\omega_{\mathrm{ed}}$ is the eddy turn-over frequency. Comparing the two extreme cases $q=0$ and $q=1$ the timescale $\tau_{\text {ed }}$ changes by a factor of $\alpha^{2}$. Taking a typical value of $\alpha=10^{-3}$ means that these time scales differ by 6 orders of magnitude. In the next section we will see that the effect of the turbulent gas on the dust, and therefore on the dust scale height, is highly dependent on this timescale $\tau_{\text {ed }}$. For this reason knowledge about $q$ is an essential requirement.

\section{A.2. Viscosity of gas and dust}

In this paper we will assume that the value of the turbulent viscosity of the gas is basically the turbulent diffusion coefficient of the gas Eq. (A.2), which corresponds to $\mathrm{Pt}=1$. Johansen $\&$ Klahr (2005) found that the ratio between these two quantities ranges from 0.8 up to 1.6 in MRI turbulence, depending on the direction, i.e. vertical or radial.

We will also assume a certain turbulent viscosity of the dust. This quantity can be expressed as the product of a characteristic mixing length and a relative turbulent velocity of the particles

$v_{\mathrm{d}}=L_{\mathrm{mix}} v_{\mathrm{t}}$

For Stokes numbers greater than unity the relative turbulent velocity between the dust particles is given by $v_{\mathrm{t}}=V_{0} / \mathrm{St}$ (Völk et al. 1980; Suttner \& Yorke 2001). The velocity $V_{0}$ denotes the turbulent velocity of the largest gas eddy introduced in the first sections. With the mixing length $L_{\text {mix }}=\tau_{\mathrm{s}} v_{\mathrm{t}}$ the viscosity of the dust is given by

$v_{\mathrm{d}}=\frac{D}{\mathrm{St}} \quad$ for $\quad \mathrm{St}>1$

where we used the Eqs. (A.2) and (A.10).

Now, for Stokes numbers smaller than unity the dust particles are well coupled to the gas, i.e. both disk components, gas and dust, behave more like one single fluid than two different types of matter. We have already seen that the diffusion coefficient for the dust in Eq. (A.14) matches the diffusion coefficient for the gas in the St $<1$ regime. We will assume the same behaviour with regard to the viscosity, e.g. that the dust viscosity equals the gas viscosity for small St. Considering this, the dust viscosity in both regimes, $\mathrm{St}>1$ and $\mathrm{St}<1$, is then given by (Schräpler \& Henning 2004)

$v_{\mathrm{d}}=\frac{D}{1+\mathrm{St}}$ 


\section{A.3. Self-induced turbulence}

In this paper we will vary $\alpha$ between a rather high value of 0.01 down to a rather low value of $10^{-6}$. As has been discussed by many authors (and will also be discussed below) we expect the following behaviour: For low $\alpha$ the dust sediments into a very thin midplane layer. However, there is a limit on how thin this layer can become, or in other words: how low the level of turbulence can become. Weidenschilling (1979) has shown that a shear-instability between the dust layer and the gas induces a weak, but non-negligible level of turbulence. This is called "self-induced turbulence".

In principle this self-induced turbulence can be described by the $(\alpha, q)$-formalism discussed above, as long as we allow that both $\alpha$ and $q$ depend on height above the midplane $z$ and radial distance to the star $r$. Determining the $\alpha$ and $q$ of this self-induced turbulence is a complex matter, and although enormous progress has been made in this field, until now there are still many open issues (Cuzzi et al. 1993; Weidenschilling 2006; Johansen et al. 2006a).

In this paper we will not explicitly address the issues of selfinduced turbulence: we will keep $\alpha$ and $q$ as parameters of the model (assuming that they do not depend on $z$ or $\mathrm{St}$ ). However, in Appendix B we will roughly estimate the level of self-induced turbulence to obtain a lower limit to the $\alpha$ that we are allowed to use.

\section{A.4. Stokes number}

A moving particle in a gas at rest loses a significant fraction of its momentum within a time called stopping time $\tau_{\mathrm{s}}$. This time depends on the friction between the particle and the gas. A strong friction means a small $\tau_{\mathrm{s}}$, and vice versa. The friction depends on the particle cross section $\sigma_{\mathrm{p}}=\pi a^{2}$ and, therefore, the particle radius $^{5} a$, the relative velocity $v_{\mathrm{p}}$ with respect to the gas and the properties of the gas (mainly gas density $\rho_{\mathrm{g}}$, isothermal sound speed $c_{\mathrm{s}}$ and molecular mean free path $l$ ).

For particles, with a size smaller than the molecular mean free path, the friction can be expressed by a simple formula:

$F_{\mathrm{e}}=\frac{4}{3} \rho_{\mathrm{g}} c_{\mathrm{s}} \sigma_{\mathrm{p}} v_{\mathrm{p}}$

This is the "Epstein drag law". In this regime the stopping time equals:

$\tau_{\mathrm{s}}=\frac{m_{\mathrm{p}} v_{\mathrm{p}}}{F_{\mathrm{e}}} \stackrel{\text { Ep. }}{=} \frac{\rho_{\mathrm{s}} a}{\rho_{\mathrm{g}} c_{\mathrm{s}}}$,

where $m_{\mathrm{p}}$ is the particle mass, which can be expressed with the particle material density $\rho_{\mathrm{s}}$ as $m_{\mathrm{p}}=(4 \pi / 3) \rho_{\mathrm{s}} a^{3}$.

For particles larger than the mean free path the drag law is much more complex. This regime is characterized by the "Stokes drag law". In this paper we focus on particles that are always smaller than the mean free path and can ignore the Stokes regime.

If the stopping time $\tau_{\mathrm{s}}$ is much smaller than the turn-overeddy time $\tau_{\text {ed }}$, the particles are strongly coupled to the gas having the same motions and the same behaviour with regard to diffusion. When $\tau_{\mathrm{s}}$ exceeds $\tau_{\text {ed }}$ by far, the dust decouples from the gas and is hardly influenced by the turbulence of the gas.

The stopping time characterizes the dynamic properties of the particle as it moves through the disk. Therefore, we can replace all microphysical particle properties like $a, \rho_{\mathrm{s}}, m_{\mathrm{p}}$ by $\tau_{\mathrm{s}}$.

\footnotetext{
${ }^{5}$ We will always assume the particles to be spherical.
}

Particles with vastly different properties (e.g. size), but the same $\tau_{\mathrm{s}}$ behave entirely the same.

Instead of using the stopping time $\tau_{\mathrm{s}}$, an even more convenient parameter is the so-called "Stokes number" $\mathrm{St}_{\mathrm{L}}$. It is defined by:

$\mathrm{St}_{\mathrm{L}}=\frac{\tau_{\mathrm{s}}}{\tau_{\mathrm{ed}}}=\tau_{\mathrm{s}} \Omega_{k} \alpha^{2 q-1}$

The particles are strongly coupled for $\mathrm{St}_{\mathrm{L}} \ll 1$ and hardly influenced by the gas for $\mathrm{St}_{\mathrm{L}} \gg 1$. For the case $q=1 / 2$ the Stokes number does not depend on $\alpha$. We will now introduce the Stokes number St by $\mathrm{St}=\mathrm{St}_{\mathrm{L}}(q=1 / 2)=\tau_{\mathrm{s}} \Omega_{k}$ and we will formulate all the particle properties in terms of $\mathrm{St}$ and $\mathrm{St}_{\mathrm{L}}$.

\section{A.5. Thickness of the dust layer}

Let us now turn to the calculation of the thickness of the dust layer. This thickness is determined by an equilibrium between dust which settles towards the midplane and diffusion which stirrs the dust up again (Dubrulle et al. 1995; Schräpler \& Henning 2004; Dullemond \& Dominik 2004b). The settling can be described by the equation of motion for a dust particle,

$\ddot{z}=-\Omega_{\mathrm{k}}^{2} z-\frac{1}{\tau_{\mathrm{s}}} \dot{z}$

For $\mathrm{St}>1 / 2$ the particle describes a damped oscillation around the midplane, corresponding to an orbit inclined with respect to the midplane. For $\mathrm{St} \ll 1 / 2$ the particle is so well bound to the gas, that the downward motion equals to an equilibrium settling motion with magnitude:

$v_{\mathrm{sett}}=-z \mathrm{St} \Omega_{\mathrm{k}}$

so that the second order differential equation Eq. (A.11) reduces to a first order differential equation:

$\dot{z}=-v_{\text {sett }}$.

The diffusion of the dust is characterized by

$D_{\mathrm{d}}=\frac{D}{1+\mathrm{St}_{\mathrm{L}}}$

which is the diffusion coefficient of the gas corrected by a factor including the coupling between dust and gas. This expression decreases with higher Stokes number since the dust is not longer affected by the gas. From the numbers $D_{\mathrm{d}}$ and $\tau_{\text {sett }}$, representing settling and diffusion, we can construct a length scale by $h^{2}=$ $D_{\mathrm{d}} \tau_{\text {sett }}$. This leads to the expression for the dust layer thickness:

$h^{2}=D_{\mathrm{d}} \max \left(\tau_{\mathrm{sett}}, 1 / 2 \Omega_{\mathrm{k}}\right)$

We resticted the settling time scale to be at least half an orbital time scale. For St larger than unity the motion of a dust particle above the midplane corresponds to a damped inclined orbit. The velocity towards the midplane of the disk can not be higher than the projected Kepler velocity $V_{\mathrm{k}} z / r$ and, hence, the time for the particle to cross the midplane not significantly smaller than an orbital time scale. However, the dynamics of bodies in quasiKeplerian orbits in turbulence may be not well described by diffusion, and the authors are aware that the approach Eq. (A.15) should be used with caution. Also the vertical distribution of the dust for the case of inclined orbits (i.e. for large Stokes number) is not Gaussian but rather bimodal, but we will ignore this effect. 
With Eqs. (A.2, A.14), as well as the expression for the gas scale height $H=c_{\mathrm{s}} / \Omega_{\mathrm{k}}$, one can rewrite Eq. (A.15) as:

$\left(\frac{h}{H}\right)^{2}=\frac{\alpha}{\min (\mathrm{St}, 1 / 2)\left(1+\mathrm{St}_{\mathrm{L}}\right)}$.

This is the most general description of the dust layer. The Stokes numbers $\mathrm{St}$ and $\mathrm{St}_{\mathrm{L}}$ allow us to calculate the thickness of the dust layer and, therefore, the dust mass densities. With these densities we are able to calculate radial drift velocities to estimate particle radial drift times in the outer parts of the disk.

\section{A.6. Gas and dust density}

With the results of the last section we now present the mass densities of gas and dust. We assume the disk to be isothermal in the $z$-direction and we use a thin disk approximation: $z \ll r$. The vertical distribution of the gas is then given by

$\rho_{\mathrm{g}}=\frac{\Sigma_{\mathrm{g}}}{\sqrt{2 \pi} H} \exp \left(-\frac{1}{2} \frac{z^{2}}{H^{2}}\right)$.

The scale height of the gas $H$ is given by $H=c_{\mathrm{s}} / \Omega_{\mathrm{k}}$. The density distribution of the dust is

$\rho_{\mathrm{d}}=\frac{\Sigma_{\mathrm{d}}}{\sqrt{2 \pi} h} \exp \left(-\frac{1}{2} \frac{z^{2}}{h^{2}}\right)$,

wherein $h$ denotes the scale height of the dust we estimated in the last section and $\Sigma_{\mathrm{d}}$ ist given by $\epsilon_{0} \Sigma_{\mathrm{g}}$. The quantity $\epsilon_{0}$ in this expression is the initial dust-to-gas mass ratio and it is usually set to 0.01 . Further investigations will show that the drift time scales depend strongly on this parameter which means that $\epsilon_{0}$ is one of the main quantities describing our system. Let us assume that the surface density $\Sigma_{\mathrm{g}}$ of the gas follows a power law

$\Sigma_{\mathrm{g}}(r)=\Sigma_{0}\left(\frac{r_{0}}{r}\right)^{\delta}$

and the temperature $T$ does so as well:

$T(r)=T_{0}\left(\frac{r_{0}}{r}\right)^{\xi}$.

We choose the power law index $\delta$ to be 0.8 following Kitamura et al. (2002). Moreover, we assume the temperature $T_{0}$ to be $200 \mathrm{~K}$, corresponding to a passive disk irradiated under an angle of 0.05 degrees around a T Tauri star with a surface temperature of $4000 \mathrm{~K}$ and $R_{\star}=2.5 R_{\odot}$. The power law index of the temperature $\xi$ is set to be $1 / 2$. The mass of the disk is given by

$M_{\mathrm{disk}}=\int_{r_{\mathrm{ev}}}^{r_{\max }} \Sigma_{\mathrm{g}}(r) r \mathrm{~d} r \mathrm{~d} \varphi=\frac{2 \pi \Sigma_{0} r_{0}^{\delta}}{2-\delta}\left[r_{\max }^{2-\delta}-r_{\mathrm{ev}}^{2-\delta}\right]$,

which we take as a parameter of our models. The size of the disk $r_{\max }$ is set to be $150 \mathrm{AU}$ following the observational results of the Taurus-Auriga region by Rodmann et al. (2006). The dust evaporation radius $r_{\mathrm{ev}}$ is $0.03 \mathrm{AU}$. Throughout the paper we will give the mass of the disk in terms of the central stellar mass $M_{\star}$ which we assume to be $1 M_{\odot}$.

If we consider Herbig Ae/Be stars instead of T Tauri stars then the disk mass and the outer disk radius increases (Natta 2004). Herbig stars are usually a factor of 4 heavier and have outer disk radii of $300 \mathrm{AU}$ and more. However, Eq. (A.21) shows that the surface density $\Sigma_{0}$ is hardly affected by this change. The disk is more massive, but since the disk extent increases as well the actual amount of mass per unit area in the disk does not change.

In this paper we use a standard velocity $v_{\mathrm{N}}$. This quantity is the velocity by which the gas moves azimuthally slower than Keplerian velocity $V_{\mathrm{k}}$, i.e. $v_{\text {gas }}=V_{\mathrm{k}}-v_{\mathrm{N}}$. It is given by (Weidenschilling 1977; Nakagawa et al. 1986)

$v_{\mathrm{N}}=-\frac{\partial_{r} p_{\mathrm{g}}}{2 \rho_{\mathrm{g}} \Omega_{\mathrm{k}}}=\frac{c_{\mathrm{s}}^{2}}{2 V_{\mathrm{k}}}\left(\frac{3}{2}+\frac{\xi}{2}+\delta\right)$

\section{Appendix B: Self-induced turbulence}

There is a limit of how thin the dust layer can be, i.e. how low the level of turbulence can become. The calculations in this paper are physically realistic as long as the turbulence parameter $\alpha$ does not drop below this value. In this part of the appendix we present the lowest possible amount of turbulence in terms of the turbulence parameterisation $\alpha$ and $q$.

The reason for a minimal amount of turbulence is the following: when the dust settles towards the midplane and the dust-to-gas ratio exceeds unity the gas is carried along with the dust. Since the gas above the midplane still moves somewhat sub-keplerian the resulting shear can destabilize the layer causing turbulence. This instability is called Kelvin-Helmholtz instability. The turbulence in turn again diffuses the particles until a steady state is reached.

In the next two subsections we will translate this effect into the $\alpha$-prescription. To do this, we have to consider two different cases. The Stokes number $\mathrm{St}_{\mathrm{c}}$ which divides these two cases is roughly given by $10^{-2}$ (Cuzzi \& Weidenschilling 2006). Let us first focus on the $\mathrm{St}<\mathrm{St}_{\mathrm{c}}$ case.

\section{B.1. St $<S t_{c}$}

In this regime of small particles the thickness of the layer is almost independent of particle properties and given by (Sekiya 1998)

$\frac{h}{H}=\sqrt{\mathrm{Ri}} \frac{H}{R}$.

The quantity $\mathrm{Ri}$ is the critical Richardson number. This number, which is usually assumed to be constant, is approximately given by $\mathrm{Ri}=0.25$ (Chandrasekhar 1961). Recent publications call this assumption in question (Gómez \& Ostriker 2005) and show that the Richardson-number required for marginal instability is eventually higher than the traditional value of 0.25 depending on the Stokes number. However, since the results of our simulations turned out to be hardly dependend on Ri this effect may be neglected in the context of radial drift velocities. A comparison between (B.1) and (A.16) yields the turbulent $\alpha$ parameter for this case.

$\alpha=\beta \operatorname{RiSt}\left(1+\mathrm{St}_{\mathrm{L}}\right)$

The quantity $\beta$ is simply the squared ratio between the isothermal sound speed $c_{\mathrm{s}}$ and the Kepler velocity $V_{\mathrm{k}}$. At $100 \mathrm{AU}$ the quantity $\beta$ is roughly of the order of $10^{-2}$. This leads to a turbulent $\alpha$ value which is at most of the order of $\alpha \sim 10^{-5}$ assuming $q=1 / 2$ and $\mathrm{Ri}=1 / 4$.

\section{B.2. $S t>S t_{c}$}

In this second regime the diffusion coefficient in self-induced turbulence for the gas is given by $D=\left(\beta V_{\mathrm{k}}\right)^{2} / \Omega_{\mathrm{k}} \mathrm{Ro}^{2}$ 
Table B.1. Variables used in the course of this paper.

\begin{tabular}{|c|c|}
\hline Variable & Explanation \\
\hline$a$ & radius of the dust particle \\
\hline$m_{\mathrm{p}}$ & mass of the dust particle \\
\hline$\sigma_{\mathrm{p}}$ & geometrical cross section of the dust particle \\
\hline$v_{\mathrm{p}}$ & velocity of the dust particle \\
\hline$f$ & filling factor of the dust particle \\
\hline$r$ & distance to the central star from a point in the midplane \\
\hline$z$ & height above the midplane \\
\hline$c_{\mathrm{s}}$ & isothermal soundspeed \\
\hline$\Omega_{\mathrm{k}}, V_{\mathrm{k}}$ & Kepler frequency, Kepler velocity \\
\hline$H=c_{\mathrm{s}} / \Omega_{\mathrm{k}}$ & gas scale height \\
\hline$h$ & dust scale height \\
\hline$\Sigma_{\mathrm{g}}, \Sigma_{\mathrm{d}}$ & surface density of the gas and the dust \\
\hline$\rho_{\mathrm{g}}, \rho_{\mathrm{d}}$ & gas and dust density \\
\hline$p_{\mathrm{g}}=c_{\mathrm{s}}^{2} \rho_{\mathrm{g}}$ & gas pressure \\
\hline$\epsilon_{0}, \epsilon$ & initial and current dust-to-gas ratio \\
\hline$\psi=1 /(1+\epsilon)$ & reduction parameter in the $\mathrm{NSH}$ solution \\
\hline$\eta=-\partial_{\mathrm{r}} p_{\mathrm{g}} / 2 \rho_{\mathrm{g}} r \Omega_{\mathrm{k}}^{2}$ & ratio between radial pressure force and gravitational force \\
\hline$r_{\mathrm{ev}}$ & evaporation radius \\
\hline$r_{\max }$ & outer radius of the disk \\
\hline$M_{\star}$ & mass of the central star \\
\hline$M_{\text {disk }}$ & mass of the disk \\
\hline$v_{\mathrm{N}}=\eta V_{\mathrm{k}}$ & typical radial drift scale \\
\hline$\alpha, q$ & turbulence parameter \\
\hline$\tau_{\mathrm{s}}$ & stopping time of the dust particle \\
\hline $\mathrm{St}=\tau_{\mathrm{s}} \Omega_{\mathrm{k}}$ & Stokes number of the particle \\
\hline $\mathrm{St}_{\mathrm{L}}=\mathrm{St} \alpha^{2 q-1}$ & modified Stokes number \\
\hline$L_{0}, V_{0}, \omega_{\mathrm{ed}}, \tau_{\mathrm{ed}}$ & length, velocity, frequency scale and time of the turbulent gas motions \\
\hline$D=v_{\mathrm{g}}, D_{\mathrm{d}}=v_{\mathrm{d}}$ & diffusion coefficients/viscosity of gas and dust \\
\hline $\operatorname{Re}_{\star}$ & critical Reynolds number \\
\hline$w_{r}, w_{\varphi}$ & radial and azimuthal velocity in the single particle drift equations \\
\hline$u^{\mathrm{NSH}}$ & radial velocity of the dust in the NSH solution \\
\hline $\bar{u}^{\mathrm{NSH}}$ & vertically integrated radial velocity of the dust in the NSH solution \\
\hline$u_{\mathrm{r}}, u_{\varphi}$ & radial and azimuthal velocity of the gas (solution of the NSe) \\
\hline$w_{\mathrm{r}}, w_{\varphi}$ & radial and azimuthal velocity of the dust (solution of the NSe) \\
\hline $\bar{u}^{\mathrm{V}}$ & vertically integrated radial velocity of the dust (solution of the NSe) \\
\hline$\Delta$ & vertical lenght scale in which dust and gas effect each other (NSe) \\
\hline$\Delta_{E}$ & lenght scale of the classical Ekman layer \\
\hline$\delta_{\mathrm{p}}$ & drift induced by the inclusion of viscosity \\
\hline
\end{tabular}

(Cuzzi \& Weidenschilling 2006). A direct comparison between the last equation and Eq. (A.2) shows that the turbulence parameter is a constant:

$\alpha=\frac{\beta}{\mathrm{Ro}^{2}}$.

To estimate the $q$ parameter we have to focus on the socalled Rossby number Ro that represents the ratio between the turn-over-frequency of the largest eddie $\omega_{\mathrm{ed}}$ and the Kepler-frequency, so that $\omega_{\text {ed }}=\Omega_{k}$ Ro. With this relation and Eq. (A.4) the turbulence parameter $q$ can be calculated to be

$q=\frac{1}{2}\left[1-\log _{\alpha}\left(\frac{2 \pi}{\mathrm{Ro}}\right)\right]$.
Taking Ro $=25$ we get $q=0.45$ which is quite close to the value 0.5 . If we assume a $\beta$ of about $10^{-2}$ at $100 \mathrm{AU}$ then the self-induced turbulent $\alpha$ parameter in this case is roughly given by $10^{-5}$. According to Cuzzi \& Weidenschilling (2006), the value of the Rossby number is uncertain up to a factor of 2-3. This means that the $\alpha$-value could be even lower and also in the order of $\sim 10^{-6}$ at $100 \mathrm{AU}$ in the disk. This might be also the case if higher stellar masses or lower temperatures are considered. 